\title{
Kontinuität und Veränderung des IAB-Betriebspanels
}

\author{
Lutz Bellmann
}

Online publiziert: 18. September 2013

(C) Institut für Arbeitsmarkt- und Berufsforschung 2013

Zusammenfassung Der Erfolg des IAB-Betriebspanels lässt sich zum einen auf den für Panelanalysen erforderlichen hohen Rücklauf und die exzellente Datenqualität sowie die Möglichkeit der Nutzung der Betriebsdatei der Beschäftigtenstatistik der Bundesagentur für Arbeit (BA) als Ziehungsgrundlage für die Stichprobe und den damit vorgenommenen Aufbau von Linked-Employer-Employee Datensätzen zurückführen. Ebenso wichtig ist die dynamische Entwicklung des Fragebogens und die Berücksichtigung neuer Fragen im IAB-Betriebpanel. Dies sind vor allem die Themenbereiche (1) betriebliche Flexibilität, (2) Fachkräftebedarf, betriebliche Aus- und Weiterbildung, (3) Innovationen sowie technischer und organisatorischer Wandel, (4) industrielle Beziehungen sowie (5) die Auswirkungen der Finanz- und Wirtschaftskrise 2008/2009 auf den Arbeitsmarkt.

\section{The development of the IAB establishment panel}

\begin{abstract}
On the one side the success of the IAB Establishment Panel Survey can be traced to the high response rate and the excellent data quality necessary for panel analyses as well as the unique possibility to use the establishment register of the Employment Statistics of the Federal Employment Agency to draw the samples and to build linkedemployer-employee data sets. Of equal importance is the dynamic development of the questionnaire and the inclusion of new questions. The most relevant topics are: (1) the establishments' flexibility, (2) the demand of skilled personnel, vocational and further training, (3) innovations as well as
\end{abstract}

Prof. Dr. L. Bellmann $(\varangle)$

IAB, Nürnberg, Deutschland

e-mail: lutz.bellmann@iab.de technological and organizational change, (4) industrial relations, and (5) the labour market effects of the Great Recession 2008/2009.

Keywords Data collection · Labor demand · Industrial relations

JEL Classification C81 · J21 · J23 · J24 · J53

\section{Einleitung}

Warum entwickelte sich das Projekt IAB-Betriebspanel (Bellmann 1997, 2002; Kölling 2000; Fischer et al. 2009) auch aus internationaler Perspektive betrachtet als eines der erfolgreichsten der empirischen Arbeitsmarkt- und Sozialforschung? Ein wichtiger Indikator für die große Resonanz in der Wissenschaft und der Fachöffentlichkeit sind die bis zum Zeitpunkt der Fertigstellung dieses Beitrags in SSCIJournals mit den Daten des IAB-Betriebpanels und des darauf aufbauenden Linked-Employer-Employee Datensatzes des IAB (LIAB) publizierten 106 Artikel. Dafür lassen sich sechs wichtige Gründe anführen.

Erstens ist die dynamische Entwicklung des Fragebogens und die Berücksichtigung neuer Fragen als Schlüssel für den Erfolg des IAB-Betriebspanels zu nennen: Der ersten Befragungswelle gingen intensive Vorbereitungen voraus, u.a. wurden verhältnismäßig umfangreiche Vorerhebungen durchgeführt (Bellmann et al. 1991 und Dostal et al. 1993) und Expertisen über bereits bestehende Betriebsbefragungen eingeholt (Gerlach et al. 1990; Hauptmanns und Ostendorf 1994). Wir haben dann ein Verfahren entwickelt, in dem neue Fragen für das IAB-Betriebspanel vorgeschlagen, im Plenum der Vertreter der am IAB-Betriebspanel beteiligten Institutionen diskutiert, in der Fragebogengruppe 
konkretisiert, im Pretest überprüft und erneut im Plenum beraten werden. Damit berücksichtigen wir den Sachverstand nicht nur der beteiligten Wissenschaftler, sondern auch den der Mitarbeiter der Ministerien und von TNS Infratest Sozialforschung, die verantwortlich für die Feldarbeit sind und ihre Erfahrungen einbringen.

Zweitens ist der hohe Rücklauf von ca. $85 \%$ bei den wiederholt befragten Betrieben als Basis für die Längsschnittanalysen hervorzuheben. Dazu hat neben den persönlichmündlichen Interviews die Unterstützung der Bundesvereinigung der deutschen Arbeitgeberverbände (BDA) und der Bundesagentur für Arbeit (BA) durch entsprechende Empfehlungsschreiben des Präsidenten bzw. des Vorstandsvorsitzenden beigetragen. Die hohe Qualität des Fragebogens ist an dieser Stelle ebenfalls als sehr wichtig anzusehen.

Drittens ist die BA Teil des deutschen Sozialversicherungssystems, die aus den über die sozialversicherungspflichtig Beschäftigten vorliegenden Daten eine Betriebsdatei aufbaut, die sich als eine hervorragende Ziehungsgrundlage für die Stichprobe der zu befragenden Betriebe bewährt hat.

Einzigartig ist viertens die Verknüpfung der Daten des IAB-Betriebspanels mit den administrativen Daten der BA. Zwar wurden Linked-Employer-Employee Datensätze in den letzten zehn Jahren in vielen Ländern aufgebaut, leider sind aber die Informationen über die Betriebe oftmals auf wenige Variablen beschränkt (Alda et al. 2005; Hamermesh 1999; Bellmann et al. 1999; Bellmann und Schank 2000; Bellmann et al. 2002a, 2002b). An dieser Stelle ist die erfolgreiche Zusammenarbeit mit dem Forschungsdatenzentrum (FDZ) besonders hervorzuheben (Allmendinger und Kohlmann 2005; Kohlmann 2005; Bender und Heining 2011; Bender et al. 2013).

Dieses Matching, aber auch die persönlich-mündlichen Interviews und das Editing bei TNS Infratest Sozialforschung erlauben Datenprüfungen und Konsistenzchecks, die dazu beitragen, die hohe Datenqualität sicherzustellen und noch zu steigern. Zudem existiert sechstens beim IAB-Betriebspanel und dem LIAB ein geregelter Zugang für externe Wissenschaftler - insofern befindet sich das IAB-Betriebspanel in guter Gesellschaft, z.B. mit dem SOEP, dem PASS oder den KombiFiD-Daten. Das IABBetriebspanel ist mit 40 neuen Projekten pro Jahr der zentrale Datensatz des FDZ. Allein der LIAB wurde im Jahr 2012 500-mal und das IAB-Betriebspanel über 900-mal über Datenfernverarbeitung abgefragt (Bender et al. 2013).

Ich kann und will an dieser Stelle nicht auf alle angesprochenen Punkte im Detail eingehen. Ich werde den Schwerpunkt meiner Ausführungen auf den ersten Punkt, also die Entwicklung des Fragebogens und die Aufnahme neuer Fragen im IAB-Betriebspanel, legen, die einerseits in der wissenschaftlichen Diskussion im In- und Ausland sowie der Fachöffentlichkeit virulent sind, aber andererseits
Forschungslücken schließen sollen, indem wir neue Themen angehen und neue Fragen stellen. Weil Fragen dazu sowohl in der amtlichen Statistik aber auch in anderen Wissenschaftlern zugänglichen Datenquellen fehlen, sind die Fragen im IAB zur betrieblichen Gewinn- und Kapitalbeteiligung, der Existenz eines Betriebsrats, der Orientierung an Tarifverträgen, der Verbreitung betrieblicher Bündnisse für Beschäftigungs- und Standortsicherung und zu den personalpolitischen Maßnahmen für ältere Arbeitnehmer einzigartig.

Folgende fünf Themenbereiche stehen im Zentrum der wissenschaftlichen Untersuchungen mit den Daten des IABBetriebspanels:

- Betriebliche Flexibilität

- Fachkräftebedarf, betriebliche Aus- und Weiterbildung

- Innovationen sowie technischer und organisatorischer Wandel

- Industrielle Beziehungen sowie

- die Auswirkungen der Finanz- und Wirtschaftskrise 2008/ 2009 auf den Arbeitsmarkt.

Diese Themenbereiche unterliegen selber natürlich einem stetigen Wandel, einige Fragen waren von Beginn an im IAB-Betriebspanel enthalten, andere kamen später hinzu: Sie wurden aber nicht nur für sich untersucht, sondern auch im Hinblick auf Beschäftigungs-, Lohn- und Produktivitätseffekte und es bestehen natürlich auch Beziehungen zwischen diesen Einflussfaktoren und Effekten. Darauf werde ich am Schluss meiner Ausführungen eingehen. Zunächst möchte ich die wichtigsten Ergebnisse auf die von uns gestellten Fragen und die Entwicklung der Fragen in diesen Themenbereichen erörtern.

\section{Betriebliche Flexibilität}

Durch die Globalisierung aber auch durch die Verbreitung moderner Informations- und Kommunikationstechnologien hat der Wettbewerb, dem sich Unternehmen und Betriebe stellen müssen, in den letzten 30 Jahren stark zugenommen. Diese Entwicklung wird sich mit Sicherheit noch weiter fortsetzen. Damit steigt die Notwendigkeit für die Betriebe, sich an die Entwicklungen auf den Beschaffungs- und Absatzmärkten flexibel anzupassen.

Ihre wissenschaftliche Reputation hat sich die Projektgruppe IAB-Betriebspanel wesentlich durch ihren Beitrag zum OECD-Projekt „Flexible Enterprise“ aufgebaut: der Projektbericht wurde als 200. Band der IAB-Reihe „Beiträge zur Arbeitsmarkt- und Berufsforschung“ im Jahre 1996 veröffentlicht. Ausgangspunkt war die Einschätzung der European Commission (1995), dass ein enger Zusammenhang zwischen der externen und internen Flexibilität eines Landes einerseits sowie seiner Wettbewerbsfähigkeit, seinem wirtschaftlichen Wachstum und dem Beschäftigungsniveau auf der anderen Seite besteht. Auch die ILO (1995) hat die Rolle 
der Unternehmen als flexible Akteure hervorgehoben, wenn es gilt, sich neuen Märkten, Technologien, Organisationsund Beschäftigungsformen anzupassen und durch Modernisierung mit vollwertigen Arbeitsplätzen produktiv und damit wettbewerbsfähig zu bleiben. Die OECD Jobs Study (1994) hat die anhaltend hohe Arbeitslosigkeit ebenfalls u.a. auf die unzureichende Flexibilität der Unternehmen zurückgeführt, sich rasch und innovativ an den Strukturwandel einzustellen. Um dieser Flexibilitätsdiskussion ein empirisches Fundament zu geben, haben die genannten Institutionen ihre Argumentationen auf Berichtssysteme auf Unternehmensebene gestützt, in Deutschland auf das IAB-Betriebspanel.

Ausgangspunkt war das Konzept von Atkinson (1985), das dem OECD-Bericht zur „Labour Flexibility“ in Enterprises (1989) zugrunde liegt. Die Zuordnung einzelner Betriebe und ihrer Beschäftigten erfolgte auf die Frage im IAB-Betriebspanel an die Betriebe nach ihrer Anpassung bei saisonalen oder sonstigen Nachfrage- bzw. Produktionsveränderungen. 1993 traf diese Situation für etwa die Hälfte aller Betriebe zu. Die intern-numerische Flexibilität, die sich neben der häufigeren Nutzung von Überstunden bzw. Sonderschichten auch mithilfe der zeitlichen Verschiebung von Urlaubsmaßnahmen sowie ggf. mit der Einführung von Kurzarbeit bei größerem betrieblichen Kapazitätsanpassungsbedarf erreichen lässt, ist für westdeutsche Betriebe typisch, während die extern-numerische Flexibilität (Einstellungen/Entlassungen, befristete Arbeitsverhältnisse, Aushilfen, Arbeitnehmerüberlassung etc.) nur bei der Hälfte der Betriebe ein bedeutsames „Anpassungsmuster“ darstellte.

Bei der internen Flexibilitätsorientierung wurde in fast allen Fällen der Auf- bzw. Abbau des betrieblichen Überstundenvolumens praktiziert. Die große Reagibilität und kurzfristige Wirksamkeit, aber auch der hohe Grad an Disponibilität aus Sicht der Betriebe hatten bei schwankender Geschäftstätigkeit Überstundenarbeit zum primären Anpassungsinstrument werden lassen, bevor auch die anderen Maßnahmen ergriffen wurden. Auch vor dem Hintergrund des Rückgangs der individuellen Arbeitszeiten war für die Betriebe ihre Abstimmung mit den Betriebs- und Servicezeiten von zentraler Bedeutung. In unserem Projekt ging es aber weniger um die Länge der Betriebszeiten, sondern um Anpassungsfähigkeit und Variationsbreite. M.a.W. war das Ausmaß, mit dem die Betriebe die zuvor im einzelnen dargestellten Arbeitszeitformen zeitgleich einsetzen, von Interesse. Dabei zeigte sich bei einer beschäftigtenproportionalen Hochrechnung der Ergebnisse aus dem IAB-Betriebspanel 1995, dass Schichtarbeit in Westdeutschland im Produzierenden Gewerbe die vorherrschende Entkopplungsform darstellte, während im Dienstleistungsbereich ,versetzte Arbeitszeiten" dominierten (Bellmann et al. 1996, 45). Untersuchungen der Bestimmungsgründe der Entwicklung der Teilzeitbeschäftigung waren ebenfalls von großer Bedeutung (Bellmann und Allaart 2007; Schank 2003; Schank et al. 2009).
Die verschiedenen Arbeitszeitformen haben natürlich ihre Bedeutung für das IAB-Betriebspanel behalten; allerdings entwickelten sich Arbeitszeitkonten zum zentralen Instrument der Anpassung der Arbeitszeiten an den Arbeitsanfall (Gerner 2010). Durch den Wegfall von bezahlten und oftmals zuschlagpflichtigen Überstunden erfuhren Arbeitszeitkonten auch auf Seiten der Arbeitgeber große Akzeptanz. Für die Beschäftigten ist die bessere Vereinbarkeit von Privatleben und Beruf wichtig. Hinzu kommen bei entsprechender Ausgestaltung Möglichkeiten für längere Arbeitsunterbrechungen z.B. für Sabbaticals und Weiterbildungszeiten sowie einen vorzeitigen Ruhestand. Seit 1999 werden daher die Verbreitung und Ausgestaltung der Ausgleichszeiträume im IAB-Betriebspanel erfasst.

Zwischen 1999 und 2011 hat sich der Anteil der Betriebe mit Arbeitszeitkonten von $16 \%$ auf fast $34 \%$ erhöht (Ellguth und Kohaut 2012). Dabei stieg der Anteil der in Betrieben mit mehr als 10 Beschäftigten mit Arbeitszeitkonten auf über $50 \%$; im europäischen Vergleich erreicht Deutschland damit den vierten Platz (European Foundation for the Improvement of Living and Working Conditions 2009). Hervorzuheben ist weiterhin die hohe Dynamik hinsichtlich der Einführung und Abschaffung von Arbeitszeitkonten, die in Längsschnittanalysen ermittelt wurde (Bellmann und Gewiese 2004; Ellguth und Kohaut 2012). Seit 2002 ist der Anteil der Betriebe mit einem Ausgleichszeitraum zwischen einem halben Jahr und einem Jahr ziemlich stabil geblieben; er liegt zwischen $39 \%$ und $43 \%$. Im Unterschied dazu fiel zwischen 2002 und 2010 der Anteil der Betriebe mit einem Ausgleichszeitraum von bis zu einem halben Jahr von $28 \%$ auf $20 \%$ deutlich. Dagegen lag der Anteil der Betriebe mit einem Langzeitkonto mit einem Ausgleichszeitraum von über einem Jahr nur bei 2 bis $4 \%$ - die Tarifpartner und der Gesetzgeber haben an dieser Stelle noch das eine oder andere Hindernis aus dem Weg zu räumen. Dies fällt offenbar größeren Betrieben leichter, denn der Anteil der Betriebe mit über 500 Beschäftigten, die über ein Langzeitkonto verfügen, hat sich zwischen 2002 und 2010 von $12 \%$ auf $22 \%$ erhöht und damit mehr als verdoppelt (Ellguth und Kohaut 2012). Die anteilsmäßig größte Bedeutung als Verwendungszweck haben dabei - übrigens mit zunehmender Bedeutung - Familienzeiten (Ellguth und Kohaut 2012).

Trotz der starken Bedeutung der internen Abwicklung der betrieblichen Anpassungserfordernisse an wirtschaftliche Volatilitäten wird in der aktuellen beschäftigungspolitischen Debatte über Flexibilisierung meist auf die diversen Formen externer Flexibilisierung wie z.B. befristete Arbeitsverträge, Leiharbeit, geringfügige Beschäftigung abgestellt. Im IAB-Betriebspanel wurden entsprechende Fragen aufgenommen - übrigens auch zur Verbreitung von Praktika und Werkverträgen. Damit wurde eine sehr unterschiedliche Inanspruchnahme in bestimmten Betriebstypen ebenso untersucht wie z.B. die Determinanten dieser Unterschiede, die 
neben den bereits erwähnten betrieblichen Anpassungserfordernissen in der Betriebsgröße und auch in den Beschäftigungsstrukturen sowie in den tarifvertraglichen Bindungen zu suchen sind (Düll und Ellguth 1999a, 1999b; Hagen 2003; Bellmann 2004; Boockmann und Hagen 2005; Hohendanner und Bellmann 2006; Promberger 2006; Hohendanner und Janik 2008; Bellmann et al. 2009; Hohendanner und Gerner 2010).

Da die zunehmende Spaltung der Gesellschaft nicht nur in Deutschland, sondern auch in anderen Ländern zum zentralen Thema avanciert ist, haben unsere Analysen zur Verbreitung befristeter Beschäftigungsverhältnisse bei Neueinstellungen sowie die Übernahme von befristet Beschäftigten in unbefristete Beschäftigungsverhältnissen (Hohendanner und Gerner 2010) sowie Verlängerung und Beendigung von befristeten Beschäftigungsverhältnissen große Aufmerksamkeit auf sich gezogen. Diese Informationen lassen sich aus keiner anderen Datenquelle ermitteln. Einzigartig sind auch die Abfragen der Gründe für den Abschluss von atypischen Beschäftigungsverhältnissen aus betrieblicher Sicht: In den Jahren 2003 und 2010 wurden dabei die Determinanten der Zeitarbeit, in den Jahren 2009 die Determinanten der befristeten Beschäftigung und der Mini-Jobs erhoben. Hinzuweisen ist auf das sehr interessante Ergebnis, dass aufgrund des hohen Anteils befristeter Neueinstellungen bei gleichzeitig geringen Übernahmequoten im öffentlichen Dienst und den sozialen Dienstleistungsbranchen gerade in diesen Branchen die Gefahr einer Verstärkung sozialer Ungleichheiten besteht (Hohendanner und Gerner 2010).

Im Rahmen eines vom BMAS geförderten Projekts zur Realisierbarkeit von Ansätzen des nachhaltigen Personalmanagements, zur Arbeitsqualität und zum wirtschaftlichen Erfolg werden in mehreren Befragungswellen an Betriebe mit mehr als 50 sozialversicherungspflichtig Beschäftigten zusätzliche Fragen gestellt. Themen sind nicht nur die Personalplanung, -beschaffung und -entwicklung, sondern auch Performance, Management, Vergütung, Bindung, Werte und Unternehmenskultur. Ausgewählte Beschäftigte (maximal 10\% der Belegschaften) in diesen Betrieben werden über persönliche Eigenschaften wie Vertrauen, Ungerechtigkeitssensibilität, Kontrollüberzeugung, Big Five und ihre Risikoeinstellung ebenso befragt wie über ihre erlebte Arbeitsqualität (u.a. Arbeitsplatzsicherheit, Work-LifeBalance, erlebte Fairness) sowie ihr Arbeitsverhalten und ihre Arbeitseinstellungen (u.a. Zufriedenheit, Committment und Engagement).

\section{Fachkräftebedarf, betriebliche Aus- und Weiterbildung}

Auch wenn das Thema Fachkräftebedarf zwischenzeitlich angesichts der Wirtschafts-, Finanz- und Schuldenkrise etwas in der wirtschafts- und arbeitsmarktpolitischen Diskus- sion in den Hintergrund getreten war, besteht große Einigkeit darüber, dass es in Zukunft wieder an Bedeutung gewinnen wird. Im Rahmen des IAB-Betriebspanels wurden seit dem Jahr 2000 immer wieder Fragen dazu gestellt. Die bisher vorliegenden Ergebnisse konnten die These eines flächendeckenden Fachkräftemangels allerdings nicht bestätigen (vgl. z.B. aktuell Dietz et al. 2012 und Bechmann et al. 2012).

An dieser Stelle soll aber nicht auf Unterschiede zwischen Berufen, Branchen und Regionen eingegangen werden. Große Aufmerksamkeit haben wir mit den Ergebnissen des IAB-Betriebspanel 2006 erzielt, in dem den teilnehmenden Betrieben Fragen zur Bedeutung verschiedener Standortfaktoren gestellt worden sind. Dabei stellte sich der Standortfaktor „Qualität des Fachkräfteangebots“ in fast allen Bundesländern als sehr bedeutsam dar: Jeweils mehr als $90 \%$ der Betriebe sagten, dass dieser Faktor eine Rolle spiele (von „,weniger wichtig“ bis ,äußerst wichtig“, für über die Hälfte der Betriebe in allen Ländern war dieser Faktor sogar „sehr wichtig“ bis ,„äußerst wichtig“, vgl. Abb. 1). Damit wird von den Arbeitgebern ein relativ einheitliches Bild von der hohen Bedeutung des Fachkräfteangebots gezeichnet. Die besten (Schul-)Noten werden für diesen Standortfaktor von den Betrieben in Sachsen-Anhalt, Thüringen und Bayern vergeben. Die schlechtesten Bewertungen erfolgten in Nordrhein-Westfalen, Schleswig-Holstein und Niedersachsen. Der Unterschied zwischen der besten und schlechtesten Bewertung liegt allerdings nur bei 0,5 Notenpunkten. Auffällig sind die überdurchschnittlich guten Bewertungen in den ostdeutschen Bundesländern. Diese liegen - mit Ausnahme Mecklenburg-Vorpommerns - oberhalb des gesamtdeutschen Durchschnitts von 2,5. Ein Grund für das positive Abschneiden der ostdeutschen Länder dürfte u.a. die aufgrund der hohen Arbeitslosigkeit relativ große Anzahl von verfügbaren Arbeitskräften für qualifizierte Tätigkeiten sein.

Im IAB-Betriebspanel werden regelmäßig verschiedene Indikatoren zur Ermittlung des Fachkräftebedarfs erhoben:

- Neueinstellungen von Fachkräften im 1. Halbjahr

- die unmittelbare Suche nach Fachkräften (zum nächstmöglichen Zeitpunkt) und

- unbesetzte Stellen für Fachkräfte.

Die Daten des IAB-Betriebspanels zeigen, dass die Nichtbesetzungsquote der Fachkräftestellen ihr bisheriges Maximum im Jahr 2000 bei einem Wert von $26 \%$ erreichte und es den meisten Betrieben auch in den Boomphasen gelang, ihren Fachkräftebedarf zu decken. Gleichwohl können unbesetzte Stellen für Fachkräfte auf betriebsindividueller Ebene insbesondere dann ein Problem darstellen, wenn es ihren wiederholt nicht gelingt, Fachkräftestellen zu besetzen. Anders gewendet macht es einen erheblichen Unterschied, ob es immer dieselben Betriebe sind, denen es nicht gelingt, ihre Fachkräftestellen zu besetzen oder ob 
Abb. 1 Durchschnittswert der einzelnen Standortfaktoren nach ihrer Bedeutung für den eigenen Betrieb, Deutschland

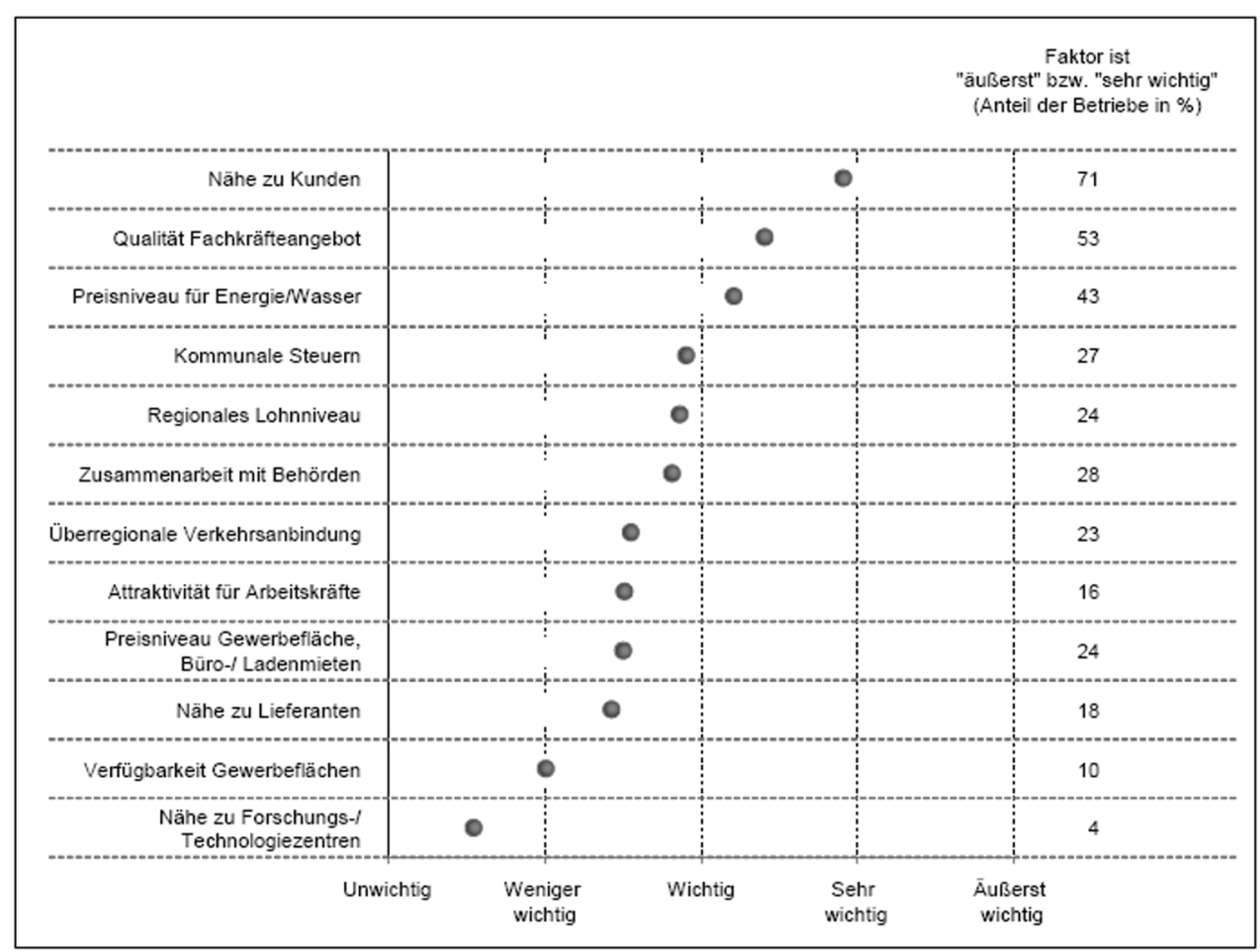

Basis: Betriebe der Privatwirtschaft

Quelle: IAB-Betriebspanel 2006 sich die Betriebe eher temporär vor Herausforderungen bei der Rekrutierung von Arbeitskräften gestellt sehen. Im Rahmen von Längsschnittanalysen lässt sich eine entsprechende Untersuchung durchführen: Für die Analyse wurden die Angaben der Betriebe, ob sie im 1. Halbjahr 2000, 2005 und 2007 auf dem externen Arbeitsmarkt nach Fachkräften suchten, verwendet (Fischer et al. 2008). Es zeigt sich, dass der überwiegende Anteil der Betriebe (61\%) zu keinen der genannten Zeitpunkte auf dem externen Arbeitsmarkt nach Fachkräften suchte, deutlich weniger Betriebe suchten dagegen gelegentlich $(29 \%)$ und lediglich $10 \%$ zu allen drei Zeitpunkten. Weitergehend wurden die Fachkräfte suchenden Betriebe danach unterschieden, ob sie die Stellen auch besetzen konnten. Dies gelang $68 \%$ der Betriebe immer, während $20 \%$ der Betriebe immer unbesetzte Stellen hatten (wenn sie Fachkräfte suchten); manchmal unbesetzte Stellen wiesen $12 \%$ der Betriebe auf.

In den Jahren 2007 und 2011 wurden die Betriebe auch gebeten, ausgehend von ihren bisherigen Erfahrungen anzugeben, welche Entwicklung sie in den nächsten Jahren erwarten. Dabei geht es einmal um den zu erwartenden Bedarf, der über Neueinstellungen gedeckt werden soll, aber auch um Stellenbesetzungen über interne Wechsel. Da den Betrieben Informationen darüber, ob Beschäftigte altersbedingt ausscheiden, relativ früh vorliegen, wurde auch eine entsprechende Frage gestellt. Anschließend wurden die Betriebe auch danach gefragt, ob und welche Schwierigkeiten sie bei der Besetzung der Stellen für Fachkräfte erwarten. Darüber hinaus wurden sie um eine Einschätzung des Ausmaßes der Beeinträchtigung gebeten. Last but not least wurden die Betriebe nach den innerbetrieblichen Maßnahmen zur Deckung des künftigen Fachkräftemangels gefragt und den Kompromissen, die sie bei der Besetzung von Fachkräftestellen eingegangen sind.

Die Abb. 2 zeigt, dass die Nutzung dieser Maßnahmen nicht zwangsläufig an konkrete Stellenbesetzungen und damit verbundene Problemlagen geknüpft sein muss, da auch die Betriebe, die keine Neueinstellungen planen, personalpolitisch durchaus aktiv sind. Die im IAB-Betriebspanel 2007 befragten Betriebe, die in den kommenden zwei Jahren Neubesetzungen von Stellen für Fachkräfte vornehmen wollen, zeigen aber ein höheres Aktivitätsniveau im Personalbereich (Fischer et al. 2008).

Ein gemeindames Ergebnis für die Betriebe mit und ohne Neubesetzungen besteht aber darin, dass der Erfahrungsaustausch sowie die betriebliche Aus- und Weiterbildung die wichtigsten Strategien darstellen, wobei die betriebliche Weiterbildung in ihrer Bedeutung noch vor der eigenen Ausbildung liegt. Diese Ergebnisse sprechen dafür, dass die älteren Arbeitnehmer immer wichtiger für die Betriebe werden: Einerseits wird die Beschäftigungsfähigkeit u.a. durch die Einbeziehung in die betriebliche Weiterbildung verbessert, andererseits werden ihre Kenntnisse und Fähigkeiten durch Wissenstransfer besser genutzt. 
Abb. 2 Personalpolitische Aktivitäten und

Fachkräftebedarf

(Mehrfachnennungen möglich)

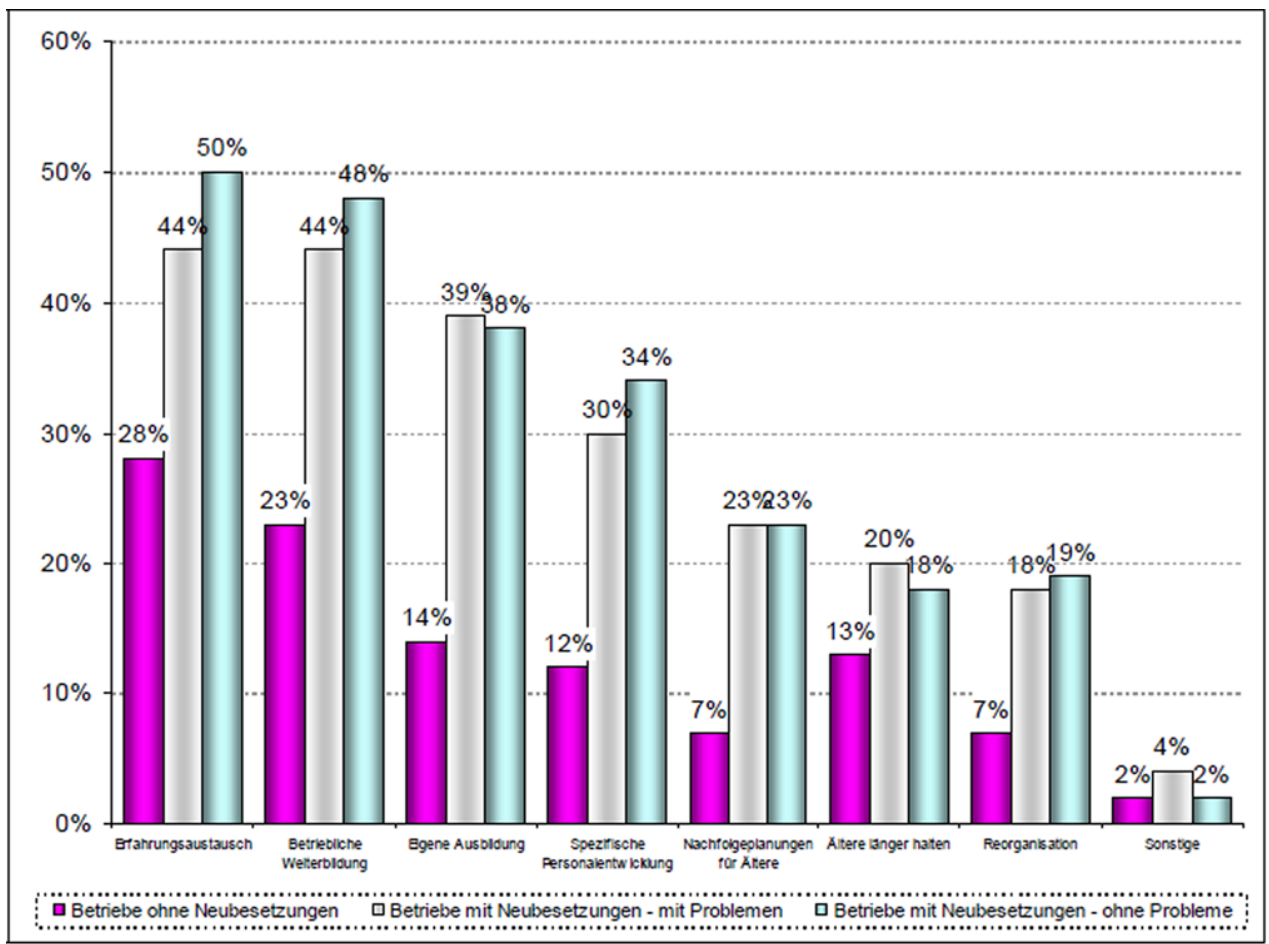

Basis: Alle Betriebe

Quelle: IAB-Betriebspanel 2007
Bis zum Aufbau des BiBB-Qualifizierungspanels im Jahr 2011 war das IAB-Betriebspanel der einzige Betriebsdatensatz, mit dem betriebliche Ausbildungsleistung abgebildet werden konnte. Dazu gehören Fragen nicht nur zur Berechtigung und Beteiligung an der betrieblichen Berufsausbildung sondern auch zur Weiterbeschäftigung von Ausbildungsabsolventen im Ausbildungsbetrieb. Insbesondere die Übernahme von Ausbildungsabsolventen im Hinblick auf den mittelfristigen Fachkräftebedarf, aber auch in Abhängigkeit von konjunkturellen Schwankungen ist z.B. von Bellmann und Hartung (2010) hervorgehoben worden und in der Abb. 3 dargestellt.

Für die Untersuchung der Einflussfaktoren wurde ein Zero-Inflated-Negative-Binomial-Regressionsmodell verwendet. Dabei zeigt sich u.a. ein hochsignifikanter Einfluss von unbesetzten Stellen für Beschäftigte mit Berufsausbildung und des erwarteten Fachkräftemangels auf die Anzahl der übernommenen Ausbildungsabsolventen. Der signifikante Effekt eines sinkenden Geschäftsvolumens sollte Anlass geben für wirtschaftlich schwierige Zeiten besondere Übergangsregelungen zu entwickeln.

Im Rahmen des IAB-Betriebspanels werden die betrieblichen Weiterbildungsaktivitäten seit 2007 jährlich erhoben; zuvor gab es einen zweijährigen Rhythmus. Im IABBetriebspanel werden Informationen zur Teilnahme an beruflicher Weiterbildung in Betrieben, die von diesen veranlasst oder zumindest teilweise finanziert wird, erhoben. Hinzu kommen Angaben für die Form der Weiterbildung (exter- ne und interne Kurse, Weiterbildung am Arbeitsplatz, Arbeitsplatzwechsel, selbst gesteuertes Lernen, Qualitätszirkel u.a.), die Teilnahme von Beschäftigten mit unterschiedlichen Qualifikationen und differenziert nach Geschlecht und Alter (Bellmann 2003; Bellmann et al. 2001; Düll und Bellmann 1998; Bellmann und Düll 1999). Anzumerken ist auch, dass im IAB-Betriebspanel Betriebe nur dann als weiterbildungsaktiv gelten, wenn sie die Weiterbildung ihrer Beschäftigten durch Freistellung und/oder Übernahme der Kosten für Weiterbildungsmaßnahmen aktiv unterstützen. Insofern ist die Definition im IAB-Betriebspanel im Unterschied zu anderen, thematisch einschlägigen Befragungen, vergleichsweise streng. Die Differenzierung der Weiterbildungsfragen nach dem Qualifikationsniveau und dem Geschlecht ist ebenso hervorzuheben wie die Frage zur Einbeziehung Älterer in betriebliche Weiterbildungsmaßnahmen und dem Angebot von an sich wünschenswerten altersspezifischen Weiterbildungsmaßnahmen. Bei letzteren ist auf die geringer Verbreitung verschiedentlich hingewiesen worden (u.a. Bellmann und Leber 2008). Multivariate Analysen zeigen aber auch, dass Betriebe dann in das Humankapital ihrer älteren Mitarbeiter investieren, wenn sie befürchten, ihren Qualifikationsbedarf auf dem externen Arbeitsmarkt nicht decken zu können (Bellmann und Leber 2008).

Weiterhin soll darauf eingegangen werden, in welchem Verhältnis berufliche Aus- und Weiterbildung stehen. Wiederholt ist darauf hingewiesen worden, dass die Weiterbildungsbereitschaft in Deutschland im internationalen Ver- 
Abb. 3 Anteil der in ein Beschäftigungsverhältnis übernommenen

Ausbildungsabsolventen (Basis:

Erfolgreiche Absolventen)

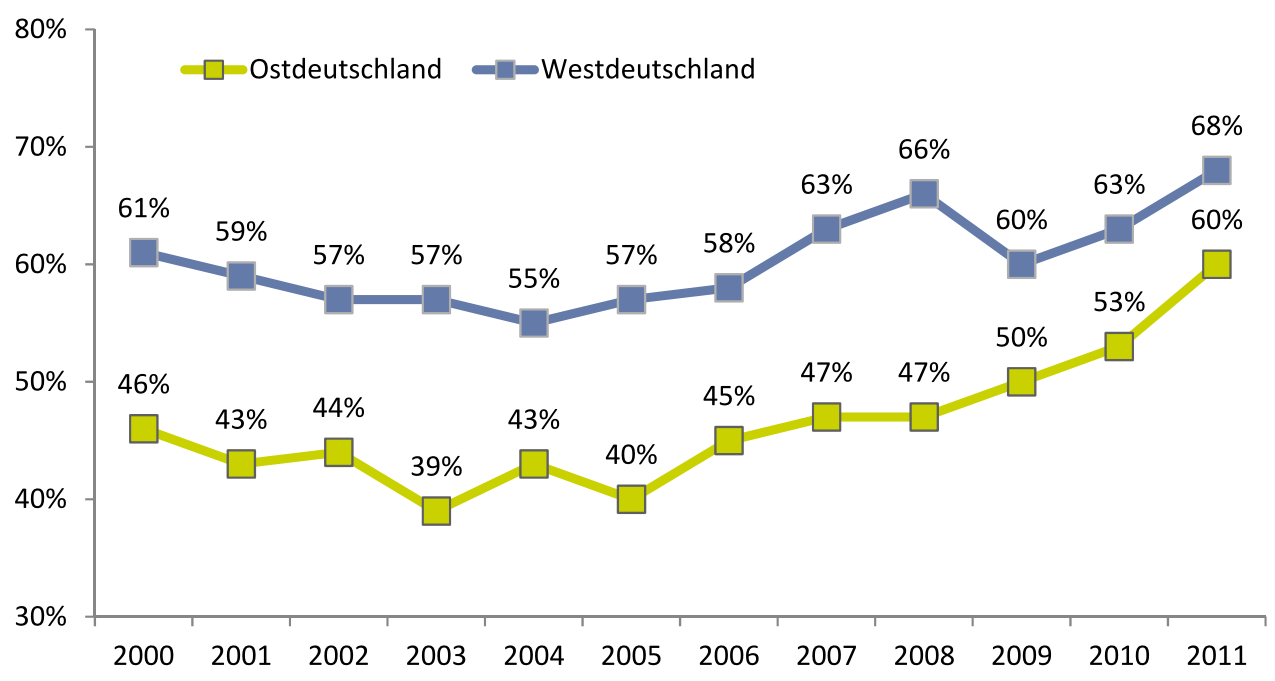

Quelle: IAB-Betriebspanel 2000 bis 2011 gleich relativ niedrig ist. Da zudem seit Jahren nur knapp ein Viertel aller Betriebe in Deutschland ausbildet (z.B. Niederalt 2004; Dietrich und Gerner 2008), haben deshalb Bellmann et al. (2010) untersucht, ob sich kleinere Betriebe häufiger auf eine der beiden Strategien beschränken (Substitutions-Hypothese), während Großbetriebe eher sowohl Aus- als auch Weiterbildung betreiben (Komplementaritäts-Hypothese). Analysen auf der Basis eines bivariaten Probitmodells zeigen jedoch, dass die Komplementaritätsstrategie insgesamt - auch über das gesamte Spektrum der Betriebsgröße - dominiert.

Abschließend soll auf die viel beachteten, auf der Basis des IAB-Betriebspanels erstellten Studien zur Einstellung und Beschäftigung älterer Arbeitnehmer, die Beurteilung ihrer Kompetenzen durch die Betriebe und das Angebot an altersspezifischen Personalmaßnahmen eingegangen werden (Bellmann et al. 2003; Bockmann und Zwick 2004; Bellmann und Leber 2008; Bellmann und Janik 2009; Brussig und Bellmann 2008; Heywood et al. 2010, 2011). Die Abb. 4 zeigt die insgesamt geringe Verbreitung von altersspezifischen Personalmaßnahmen, die zwar in größeren Betrieben stärker genutzt werden, bei denen es aber sicherlich noch „Luft nach oben“ gibt. Nach der Wirtschaftsund Finanzkrise 2008/2009 lassen sich aber durchaus sichtbare Fortschritte z.B. bei der besonderen Ausstattung der Arbeitsplätze, der Anpassung der Arbeitsanforderungen und der Einbeziehung Älterer in Weiterbildungsmaßnahmen feststellen. Weniger hat sich beim Aufbau altersgemischter Arbeitsgruppen und der altersspezifischen Weiterbildung getan. Die Rolle der Altersteilzeit ist nach wie vor dominierend; wobei ihre Bedeutung vor allem im Vergleich der Jahre 2008 und 2011 deutlich abgenommen hat.

\section{Innovationen, technischer und organisatorischer Wandel}

Die Schaffung eines gemeinsamen europäischen Wirtschaftsraumes, die Einführung des Euro und der Aufholprozess in Ostdeutschland haben das Interesse von Wissenschaft und Politik an den betrieblichen Forschungs- und Innovationsaktivitäten sowie ihrer Standortwahl, aber auch an arbeitsorganisatorischen Änderungen geweckt. Das wirtschaftliche Wachstum von Regionen, die Entwicklung von Unternehmen und dabei insbesondere ihre Wettbewerbsfähigkeit als Voraussetzung für die Schaffung von Arbeitsplätzen und die damit verbundene soziale Teilhabechancen stehen dabei im Zentrum der Aufmerksamkeit. Innovationen, technischer und organisatorischer Wandel lösen oftmals einen Qualifikationsbedarf aus und/oder führen zur Suche und ggf. Einstellung von geeigneten Fachkräften.

Traditionelle Indikatoren der Messung von Innovationen wie Forschungs- und Entwicklungsausgaben und Patente haben sicherlich ihre Vor- aber auch Nachteile. Im IABBetriebspanel wird deshalb der subjektiv-betriebliche Innovationsbegriff entsprechend des Oslo-Manuals der OECD (2005) verwendet. Dabei kommt es darauf an, ob aus Sicht des innovierenden Betriebes das Produkt oder die Dienstleistung erstmalig in das Sortiment aufgenommen wurde oder ein neuer Prozess implementiert wurde. Dabei werden im IAB-Betriebspanel drei Typen von Produktinnovation unterschieden:

(a) Verbesserung oder Weiterentwicklung eines in der Produktpalette des Betriebs vorhandenen Produktes (Weiterentwicklungen oder inkrementelle Innovation)

(b) Erweiterung der Produktpalette des Betriebs um ein bereits am Markt existierendes Produkt (Imitation) 


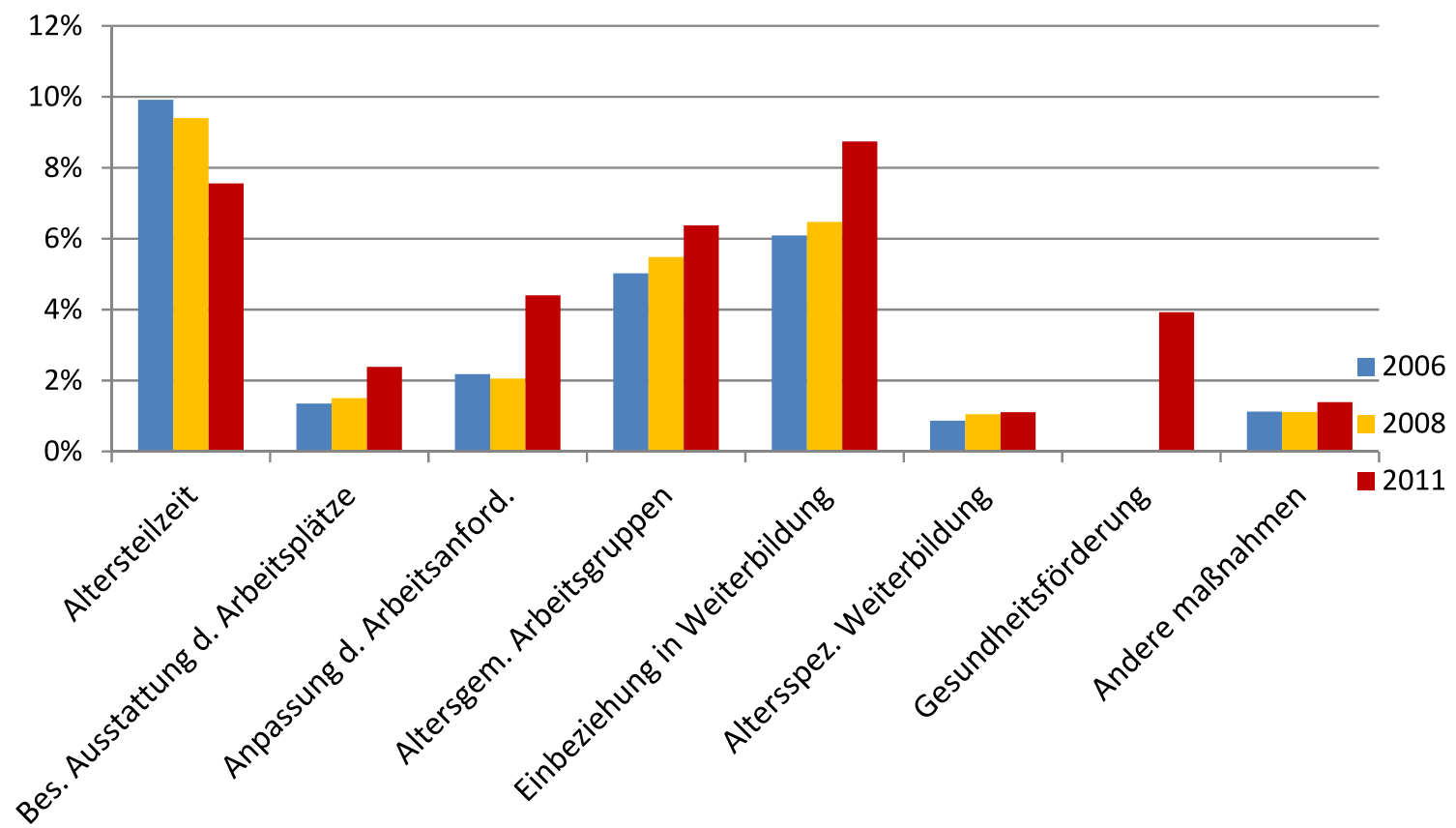

Anmerkung: Die Gesundheitsförderung wurde erstmals im Jahr 2011 erfasst.

Quelle: IAB-Betriebspanel

Abb. 4 Angebot an einzelnen altersspezifischen Personalmaßnahmen 2006-2011

(c) Einführung eines für den Betrieb völlig neuen Produktes, für das der Betrieb den Markt erschließen muss (Marktneuheit oder radikale Innovation).

Prozessinnovationen sind im Betrieb entwickelte oder eingeführte Verfahren, die den Produktionsprozess oder das Dienstleistungsangebot merklich verbessern. Die Umsetzung des technischen Fortschritts in Form von Prozessinnovationen dient in erster Linie der Effizienzsteigerung und der Qualitätsverbesserung. Wegen der großen Bedeutung der Wirtschafts- und Finanzkrise 2008/09 wurde in der 2009er Welle des IAB-Betriebspanels auch nach den Hemmnissen für die Durchführung von Prozessinnovationen gefragt. Die Abfrage des technischen Stands oder der Maschinen und Anlagen in Form einer fünfstufigen Likertskala erfolgt dagegen in jeder Welle des IAB-Betriebspanels.

Mit den Veränderungen in der Managementpraxis, die branchenübergreifend mit dem Begriff des Human Resource Management bezeichnet worden sind, sollen die zur Verfügung stehenden (Human-)Ressourcen besser genutzt, der mit der tayloristischen Organisationsform anfallende Stellen- und Schnittstellenballast minimiert und das Qualifikations- und Motivationspotential der Mitarbeiter besser gehoben werden (Womack et al. 1991; Hammer und Champy 1994). Die Erhöhung der organisationellen Flexibilität soll die Anpassung an veränderte Marktsituationen erleichtern. Erreicht wird dies in der Regel durch die Dezen- tralisierung von Kompetenz und Verantwortung, die Einführung von Gruppenarbeit und den Abbau von starren Abteilungsgrenzen zu erreichen. Damit einher geht die intensive Nutzung von Informations- und Kommunikationstechnologien. Reorganisationsmaßnahmen können nach der Änderung der betrieblichen Aufgabenstruktur der Arbeitnehmer und der betrieblichen Produktionsstruktur (die Fertigungstiefe und formale Prozeduren betreffend) unterschieden werden.

Folgende Änderungen der Produktionsstruktur wurden seit 1995 im IAB-Betriebspanel regelmäßig abgefragt:

- mehr Eigenfertigung/Eigenleistung

- mehr Zukauf von Produkten/Leistungen

- umweltbezogene organisatorische Maßnahmen (Öko-, Produkt- und Stoffbilanzen)

- Verbesserung der Qualitätssicherung sowie

- Neugestaltung der Beschaffungs- und Vertriebswege bzw. Kundenbeziehungen

Folgende Änderungen der Aufgabenstruktur werden erhoben:

- Reorganisation von Abteilungen/Funktionsbereichen

- Verlagerung von Entscheidungen nach unten

- Einführung von Gruppenarbeit/eigenverantwortlichen Arbeitsgruppen

- Einrichtung von Einheiten mit eigener Kosten-/Ergebnisermittlung 
Tab. 1 Anteil der Betriebe mit Innovation (2008) im Verarbeitenden Gewerbe West- und Ostdeutschlands nach Betriebsgrößenklasen (Anzahl Beschäftigte) - in \% (Mehrfachnennungen möglich)

\begin{tabular}{|c|c|c|c|c|c|c|c|c|c|c|}
\hline \multirow{3}{*}{$\begin{array}{l}\text { Anzahl der } \\
\text { Beschäf-tigten }\end{array}$} & \multirow{2}{*}{\multicolumn{2}{|c|}{ Innovation }} & \multicolumn{6}{|c|}{ Produktinnovation } & \multirow{2}{*}{\multicolumn{2}{|c|}{ Prozessinnovation }} \\
\hline & & & \multicolumn{2}{|c|}{ Imitationen } & \multicolumn{2}{|c|}{ Weiterentwicklungen } & \multicolumn{2}{|c|}{ Marktneuheiten } & & \\
\hline & West & Ost & West & Ost & West & Ost & West & Ost & West & Ost \\
\hline 1 bis 9 & 41,4 & 41,7 & 22,8 & 21,0 & 33,7 & 35,1 & 6,8 & 5,7 & 5,9 & 10,0 \\
\hline 10 bis 49 & 49,9 & 47,4 & 23,7 & 22,6 & 42,5 & 37,6 & 8,0 & 10,6 & 16,7 & 15,1 \\
\hline 50 bis 249 & 71,3 & 65,1 & 33,2 & 29,9 & 63,4 & 58,0 & 15,2 & 21,0 & 34,0 & 25,6 \\
\hline ab 250 & 87,1 & 80,6 & 44,0 & 41,3 & 84,3 & 73,9 & 21,0 & 22,7 & 53,3 & 47,4 \\
\hline Insgesamt & 48,1 & 46,4 & 40,7 & 38,7 & 24,6 & 22,6 & 8,3 & 9,0 & 13,4 & 13,7 \\
\hline
\end{tabular}

Quelle: Berechnungen von Günther und Marek (2011) mit den Daten des IAB-Betriebspanels 2009

Zusätzlich gab es eine Antwortmöglichkeit „,sonstige organisatorische Änderungen“.

Hervorzuheben ist das große Interesse der politischen Entscheidungsträger an international vergleichenden Analysen von technischen und organisatorischen Änderungen, die beispielsweise im Measuring the Dynamics of Organisation and Work (MEADOWS)-Projekt ihren Niederschlag gefunden hat. Im Rahmen dieses Projekts wurden Richtlinien für den Aufbau von Datensätzen zur Messung der Dynamik der Organisationsentwicklung und der Erwerbsformen auf der Betriebs- und Beschäftigtenebene entwickelt (MEADOWSProjekt 2010).

Die Verwendung von Modulen zur Erfassung von betrieblichen Innovations- und Regorganisationsaktivitäten in breiter angelegten Betriebsbefragungen dient aber auch dazu, die von ihnen ausgehenden Effekte zu ermitteln. Mit den Daten des IAB-Betriebspanels wurden die Beschäftigungswirkungen von umweltbezogenen Innovationen beispielsweise von Blien et al. (2000) sowie Horbach (2003, 2010), und die betrieblichen Produktivitätswirkungen von organisatorischen Änderungen von Möller (2000), Kölling und Möller (2002), Alda und Bellmann (2002), Wolf und Zwick (2002, 2008), Kölling und Schank (2002) untersucht. Beckmann und Bellmann (2000), Bauer und Bender (2001, 2004), Bellmann et al. (2002b, 2002a), Gerlach et al. (2002) und Bellmann und Pahnke (2006) widmen sich den Beschäftigungs- und Qualifikationseffekten und Bellmann et al. (2008) auch den Lohneffekten betrieblicher Reorganisationsmaßnahmen. Dabei bildete meistens der LIAB die Datengrundlage.

In der Tab. 1 ist der Anteil der Betriebe mit Innovationen im Verarbeiteten Gewerbe West- und Ostdeutschlands nach Betriebsgrößenklassen für das Jahr 2008 dargestellt, um die Unterschiede in der Struktur der Betriebsgröße in beiden Landesteilen zu berücksichtigen. Durchgängig nimmt der Anteil der Innovationen mit der Betriebsgröße zu. Dabei zeigt sich, dass der Anteil der Betriebe mit mindestens einer der vier betrachteten Innovationsarten in Westdeutschland bei Betrieben insgesamt und bei den Betrieben mit 10 und mehr Beschäftigten größer ist als in Ostdeutschland, bei den Kleinbetrieben schneidet Ostdeutschland aber etwas besser ab. Während bei den Produktinnovationen insgesamt und bei Weiterentwicklungen von Produkten die westdeutschen Betriebe zum Teil deutlich besser sind als die ostdeutschen Betriebe (mit Ausnahme der Betriebe mit 1 bis 9 Beschäftigten bei den Weiterentwicklungen von Produkten), liegen die ostdeutschen Betriebe bei den Marktneuheiten als der anspruchsvollsten Produktinnovationsart leicht vor den westdeutschen Betrieben (mit Ausnahme der Betriebe mit 1-9 Beschäftigten).

Bei den Prozessinnovationen ist der Anteilswert für die westdeutschen Betriebe in den Betriebsgrößenklasen 10 bis 49 Beschäftigte, 50 bis 249 Beschäftigte und mehr als 250 Beschäftigte in Westdeutschland größer, nicht aber für die Betriebe mit 1 bis 9 Beschäftigten und für die Gesamtheit aller Betriebe. Zudem finden Günther und Marek (2011) in ihrer regressionsanalytischen Untersuchung, dass die betrieblichen Weiterbildungsaktivitäten einen positiv signifikanten Einfluss auf die verschiedenen Innovationsaktivitäten in Ost-, aber nicht in Westdeutschland ausüben, selbst wenn für eine ganze Reihe von weiteren Einflussfaktoren wie die Existenz einer eigenen Forschungs- und Entwicklungsabteilung, der Modernität der Maschinen und Anlagen, den Anteil der Beschäftigten mit Hochschulabschluss, der Ertragslage, der Exportaktivität, ausländischen Kapitalbeteiligung, Wirtschaftszweigzugehörigkeit und BetriebsgröBe kontrolliert wird.

Die Rolle des regionalen Umfelds der Betriebe für ihre Innovationsaktivitäten analysieren Bellmann et al. (2013) für die Jahre 2006 bis 2010. Im Rahmen eines Drei-EbenenModells (Rabe-Hesketh und Skrondal 2012) wird berücksichtigt, dass die Regionalvariablen aggregiert vorliegen. Für die Untersuchung wurde die regionale Abgrenzung von 50 Arbeitsmarktregionen nach Kropp und Schwengler (2011) verwendet. Von den auf der regionalen Ebene gemessenen Variablen Arbeitslosenquote, Anteil von Absolventen in den Fächern Mathematik, Ingenieurwissenschaften, Naturwissenschaften und Technik (MINT) und der Nähe 
zu Forschungs- und Technologiezentren sowie Hochschulen erweisen sich die Arbeitslosenquote und der Anteil der MINT-Absolventen bei radikalen Produktinnovationen und Prozessinnovationen nur der Anteil der MINT-Absolventen als positiv und signifikant.

Die ausgesprochen schlechten Arbeitsmarktchancen der Personen ohne Bildungsabschluss werden in der Literatur oftmals mit dem technologischen und dem organisatorischen Wandel in Verbindung gebracht (Krugman 1994). Der Anstieg des Anteils der Facharbeit für Deutschland von $69 \%$ auf $74 \%$ im Zeitraum 2001 bis 2008 kann auch als Beleg für diese These betrachtet werden (Bellmann und Stegmaier 2010). Mit dem LIAB konnten zwar die erwarteten positiven Auswirkungen des organisatorischen Wandels auf die betriebliche Arbeitsnachfrage ermittelt, aber für den ,skill bias“ des organisatorischen Wandels konnten keine eindeutigen Hinweise gefunden werden (Bellmann und Pahnke 2006). Diese Analysen erfolgten für einen verhältnismäßig langen Zeitraum von 1996 bis 2004. Dabei wurden dynamische Arbeitsnachfragefunktionen geschätzt und die Effekte einzelner betrieblicher Reorganisationsmaßnahmen betrachtet. Im Vergleich verschiedener Einzelmaßnahmen wird für West- und für Ostdeutschland deutlich, dass vor allem die Reorganisation von Abteilungen für Un- und Angelernte negative Beschäftigungseffekte zeigt. Allerdings zeigen die Ergebnisse auch, dass die Einführung von Gruppenarbeit sich durchaus positiv auf das Beschäftigungsniveau Un- und Angelernter auswirken kann.

In einem weiteren Papier wurde von Addison et al. (2008) die Nachfrage nach unterschiedlich qualifizierten Beschäftigten wiederum mit dem LIAB untersucht. Auch bei dieser Untersuchung war die empirische Evidenz hinsichtlich des „skill bias“ des organisatorischen Wandels nicht eindeutig. Allerdings wurden in Übereinstimmung mit Bellmann und Schank (2000) Lohnrigiditäten als mögliche Ursache für die hohe Arbeitslosigkeit Un- und Angelernter ermittelt.

Schließlich finden auch Bellmann et al. (2008) positive Lohneffekte und stabilisierende Beschäftigungseffekte häufiger bei Beschäftigten mit mittlerem und hohem Ausbildungsstand. Eindeutig beschäftigungsdestabilisierende Effekte für Un- und Angelernte lassen sich dagegen nicht feststellen. Insofern müssen deshalb weitere Erklärungsfaktoren für eine Diagnose der Beschäftigungs- und Entlohnungssituation unterschiedlicher Qualifikationsgruppen herangezogen werden. Dustmann et al. (2009) haben ebenfalls den LIAB genutzt, um die Lohnänderungen zwischen 1995 und $2004 \mathrm{zu}$ analysieren. Sie finden, dass die abnehmende Bindung an Flächentarifverträge und die Entwicklung der Löhne beim untersten 5-Prozent-Perzentil einen wesentlich stärkeren Einfluss haben als die Entwicklung der Löhne bei den oberen Lohngruppen. Entscheidend ist aus Sicht der Autoren die Möglichkeit der Gestaltung und Durchführung von Tätigkeiten als Routineaufgaben.

\section{Industrielle Beziehungen}

In der öffentlichen Diskussion wird den Themen „Erosion des Flächentarifvertrags“, der abnehmenden Tarifbindung und der „Stärkung der betrieblichen Aushandlungsebene“ seit etwa 20 Jahren große Aufmerksamkeit gewidmet. Die Erhebungen des IAB-Betriebspanels begann insofern in den frühen 90er Jahren gerade rechtzeitig, um diese Entwicklung umfassend abbilden zu können. Seit 1995 werden die Betriebe danach gefragt, ob sie an Branchen- und Firmentarifverträge gebunden sind und seit 1993, ob sie einen Betriebs- oder Personalrat haben. Zudem wird regelmäßig seit 1995 nach der übertariflichen Entlohnung, der Orientierung an Tarifverträgen (erstmals 1998), zur Bedeutung und zum Inhalt von tariflichen Öffnungsklauseln (erstmals 2005) sowie dem Abschluss von betrieblichen Bündnissen für Beschäftigungs- und Standortsicherung (erstmals 2006) gefragt. Auch aus internationaler Perspektive ist die Entwicklung der Tarifbindung als eines der wichtigsten Merkmale des deutschen Arbeitsmarktes beispielsweise jüngst von Card et al. (2012) unter Verwendung des LIAB hervorgehoben worden.

Zunächst ist der Rückgang der Anzahl der Beschäftigten, die in an Branchentarifverträge gebundenen Betrieben arbeiten, im gesamten Beobachtungszeitraum 1996 bis 2011 in Westdeutschland von $70 \%$ (bzw. in der Privatwirtschaft $66 \%$ ) auf $54 \%$ (bzw. $48 \%$ ) und in Ostdeutschland von $56 \%$ (bzw. $48 \%$ ) auf $37 \%$ (bzw. $30 \%$ ) beeindruckend (Ellguth und Kohaut 2012, 300). Auf der gesamtwirtschaftlichen Ebene reduzierte sich damit die Flächentarifbindung in Westdeutschland um 16 Prozentpunkte und lag damit im Jahre 2011 unter dem ostdeutschen Niveau aus dem Jahr 1996. In Ostdeutschland verlief der Erosionsprozess parallel dazu, aber noch deutlicher: Der Rückgang betrug sogar 19 Prozentpunkte.

Weiterhin stellt sich die Frage nach dem Timing des Prozesses. Die entsprechenden Auswertungen von Ellguth und Kohaut (2012) aus dem IAB-Betriebspanel zeigen, dass die jährliche Abnahme der Flächentarifbindung in Westdeutschland bis zum Jahr 2008 (mit der Ausnahme des Jahres 2002) und in Ostdeutschland bis zum Jahr 2004 jeweils mindestens 2 Prozentpunkte betrug. Seither ist eine Stabilisierung in Ostdeutschland (mit der Ausnahme des Jahres 2009) und Westdeutschland (mit einem kleinen Anstieg in den Jahren 2009 und 2010) zu beobachten. Die Analysen von Ellguth und Kohaut (2012) zeigen auch, dass die Entwicklung im öffentlichen Dienst und in den Organisationen ohne Erwerbszweck zwar in die gleiche Richtung ging, aber wesentlich moderater verlaufen ist.

Erstmals im Jahre 2003 wurden die Betriebe im IABBetriebspanel auch danach gefragt, ob sie sich hinsichtlich der Entlohnung, den Arbeitszeiten oder bestimmten Zusatzleistungen an den Regelungen eines Branchentarifvertrags 
orientieren. Interessanterweise haben sich die Anteile der Betriebe, die zwar selber nicht an einen Branchentarifvertrag gebunden sind, sich aber trotzdem bei der Entlohnung ihrer Mitarbeiter an einen solchen anlehnen, im Zeitablauf kaum verändert. Waren es $43 \%$ der Betriebe ohne Branchentarifvertrag, die im Jahre 2003 ihre Entlohnung an einem solchen orientierten, waren es 8 Jahre etwas mehr als die Hälfte der nicht tarifgebundenen Betriebe, obwohl die Bindung an einen Flächentarifvertrag in diesem Zeitraum deutlich zurückgegangen ist und der Anteil der Betriebe, die sich an einem Branchentarifvertrag orientieren, gestiegen ist (Ellguth und Kohaut 2012).

Als Antwort auf die weitergehende, den Charakter der Tariforientierung vertiefende Frage im IAB-Betriebspanel 2011, ob sie im Durchschnitt vergleichbare, höhere oder niedrigere Löhne und Gehälter bezahlen, gaben $75 \%$ der westdeutschen und sogar $83 \%$ der ostdeutschen Betriebe an, in ihrer Höhe und den Branchentarifvertraglichen festgelegten, vergleichbare Löhne zu zahlen, während $22 \%$ (8\%) der Betriebe in Westdeutschland (Ostdeutschland) sogar höhere Löhne und nur $4 \%$ (9\%) der Betriebe in Westdeutschland (Ostdeutschland) schlechter als im Branchentarifvertrag festgelegt entlohnen. Dabei wurden Anpassungen der Arbeitszeit und anderer Regelungen berücksichtigt (Ellguth und Kohaut 2012).

Die Entwicklung der betrieblichen Mitbestimmung als der zweiten tragenden Säule im bundesdeutschen System industrieller Beziehungen war Anlass für den Gesetzgeber mit der Reform des Betriebsverfassungsgesetzes 2001 gegenzusteuern: Durch die „Erleichterung der Bildung von Betriebsräten durch Entbürokratisierung des Wahlrechts" (Presse- und Informationsamt der Bundesregierung 2001) und erweiterte Mitbestimmungsrecht z.B. im Bereich der betrieblichen Weiterbildung ( $\S 96$ Betriebsverfassungsgesetz) sollte der Anteil der Beschäftigten in Betrieben, in denen ein Betriebs- oder Personalrat existiert, zumindest stabilisiert und damit eine Erosion wie bei der Flächentarifbindung vermieden werden (Kohaut und Schnabel 2003; Addison et al. 2004).

Allerdings ergaben multivariate Analysen mit den Daten des IAB-Betriebspanels für den Zeitraum 1998 bis 2005 eine signifikant geringere Verbreitung von Betriebsratsgremien für den Zeitraum nach der Reform des Betriebsratsverfassungsgesetzes 2001 (Bellmann und Ellguth 2006). Dieser Trend hat sich in den folgenden Jahren fortgesetzt: Für den Zeitraum 1996 bis 2011 haben Ellguth und Kohaut (2012) einen Rückgang beim Anteil der Beschäftigten in Betrieben mit Betriebsrat (Basis sind die Betriebe, in denen ein Betriebsrat gewählt werden kann, d.h. privatwirtschaftliche Betriebe am 5 Beschäftigte, ohne Landwirtschaft und Organisationen ohne Erwerbszweck) von $51 \%$ auf $44 \%$ in Westdeutschland und von $43 \%$ auf $36 \%$ in Ostdeutschland - also jeweils um 7 Prozentpunkte - ermittelt. Diese
Entwicklung führen die Autoren vor allem auf die Erosionsprozesse im Bereich der Betriebe in der Größenordnung von 51 bis 500 Beschäftigten zurück, also ein Segment, in dem ein Betriebsrat weder eine Selbstverständlichkeit noch eine Ausnahme darstellt, sondern aus Arbeitnehmersicht ein spezifisches Organisationsinteresse und einen Regelungsbedarf erfordert. Interessanterweise verläuft dieser Erosionsprozess in West- und Ostdeutschland parallel, wobei allerdings das niedrigere Niveau in Ostdeutschland anzumerken ist.

Das Zusammenwirken betrieblicher Mitbestimmung und der Bindung an Branchentarifverträge kann ebenfalls mit den Daten des IAB-Betriebspanels analysiert werden. Betrachtet werden einerseits die so genannten betrieblichen „Vertretungslücken“, also Betriebe, die zwar tarifgebunden sind, aber über keinen Betriebsrat verfügen, andererseits die so genannten „weißen Flecken“ in der Tarif- und Mitbestimmungslandschaft, wo es keinerlei gesetzlich legitimierte Interessenvertretung gibt. Auch hier hat es unterschiedliche Entwicklungen gegeben: Arbeiteten im Jahre 2003 noch $25 \%$ aller Beschäftigten in westdeutschen Betrieben mit Branchentarifbindung aber ohne Betriebsrat, so waren dies im Jahr 2011 nur noch $21 \%$, in Ostdeutschland stagnierte der Wert bei $15 \%$ (Ellguth 2005; Ellguth und Kohaut 2012). Im selben Zeitraum erhöhte sich der Anteil der Beschäftigten in Betrieben, die nicht an einen Branchentarifvertrag gebunden und ohne Betriebsrat waren, in Westdeutschland von 27 auf $35 \%$ deutlich, während er in Ostdeutschland von 46 auf $47 \%$ nur leicht anstieg.

\section{Auswirkungen der globalen Finanzkrise 2008/09 auf den Arbeitsmarkt}

Da wir nicht zu der kleinen Gruppe von Ökonomen gehört haben, die das Ausmaß und den Zeitpunkt des Eintritts der globalen Finanzkrise 2008/09 vorhergesehen haben, mussten wir uns zunächst für unsere Analysen auf die vorhandenen Daten des IAB-Betriebspanels auf der Basis eines nicht im Lichte eine Krise gestalteten Fragebogens konzentrieren. Gleichwohl haben wir in den Erhebungen 2009, 2010 und 2011 spezielle, sich auf die Auswirkungen der globalen Finanzkrise beziehende Fragen an die Betriebe gestellt.

Zunächst war der Rückgang des Bruttoinlandsprodukts von einem Ausmaß, das nur von der großen Depression 1929 übertroffen wurde (Stiglitz 2009). Vor diesem Hintergrund wurde die Stabilisierung des Beschäftigungsniveaus in Deutschland eine auch international wirklich beachtete Leistung. Das „German Jobs Miracle“ (Krugman 2009) war und ist dann auch Gegenstand der wissenschaftlichen Debatte, denn „Wunder“ gibt es natürlich nicht für Wissenschaftler. Obwohl der Rückgang des Bruttoinlandsprodukts der deutschen Volkswirtschaft stärker als in den USA, ähnlich wie in Schweden und etwas geringer als in Japan ausfiel, verlief die Entwicklung der Arbeitslosigkeit und der Beschäftigung weitaus günstiger. 
Ein erster Analyseschritt bestand in der Ermittlung der Beschäftigungseffekte der Krise 2008/09. Wenn als Betroffenheit von der globalen Krise eine negative erwartete Umsatzentwicklung für 2009 bei einer positiven oder zumindest gleichbleibenden erwarteten Umsatzentwicklung in 2008 angenommen wird, ergibt sich ein Beschäftigungsrückgang von $6 \%$ bei den von der Krise 2008/09 betroffenen Betrieben (Bellmann und Gerner 2011a, 192). ${ }^{1}$ Besonders stark war der Rückgang der Anzahl der Leiharbeitnehmer, der im Jahresvergleich 2008/09 um mehr als $30 \%$ sank (Bellmann und Gerner 2011a, 186). Weitergehende Analysen von Bellmann und Stegmaier (2011) haben eine überproportionale Betroffenheit von Beschäftigten in Tätigkeiten, die keine Berufsausbildung erfordern, nachgewiesen.

Die Stabilisierung des Beschäftigungsniveaus auf der Makroebene ging aber durchaus mit Beschäftigungsverlusten in der Höhe von 1-6\% in einzelnen Wirtschaftsbereichen aber auch im Produzierenden Gewerbe wie dem Maschinenbau, der Automobilindustrie, der Elektrotechnik und der chemischen Industrie einher. Nicht unerwähnt bleiben soll der Beschäftigungsaufbau in bestimmten Dienstleistungsbranchen wie dem Gesundheits- und Sozialbereich.

Im zweiten Schritt wurden die Lohneffekte berechnet. Die Erwartung bestand darin, dass gerade die besser bezahlten Beschäftigten Lohneinbußen hinnehmen mussten, weil die Krise die wirtschaftlich stärksten Branchen besonders stark getroffen hatte. Im Gegensatz dazu zeigen unsere Ergebnisse einen insignifikanten Lohneffekt (Bellmann und Gerner 2011a, 192). Allerdings handelt es sich dabei wiederum um einen aggregierten Effekt, der dadurch entsteht, dass Arbeitszeitkontensysteme die individuellen Einkommen stabilisieren: In Betrieben ohne Arbeitszeitkonten ist die Bruttolohn- und -gehaltssumme (ohne Urlaubsgeld) pro Beschäftigten krisenbedingt um 3,5\% zurückgegangen, während sie in den Betrieben mit Arbeitszeitkonten sogar leicht um 1,3\% gestiegen ist (Bellmann und Gerner 2011a, 197). ${ }^{2}$ Ein verhältnismäßig geringerer Einfluss der Arbeitszeitkonten für die Beschäftigungsstabilisierung in der globalen Krise 2008/2009 wird auch von Gerner (2012) ermittelt.

In Deutschland waren von der globalen Krise 2008/09 vor allem die exportintensiven Wirtschaftszweige des Produzierenden Gewerbes, wie der Maschinenbau, die Automobilindustrie, die Elektrotechnik und die chemische Industrie betroffen. Joachim Möller (2010) hat darauf hingewiesen, dass in diesen Sektoren bereits vor der Rezession Besetzungsprobleme bei offenen Stellen hatten und damit die Sorge vor einem künftigen Fachkräftemangel be-

\footnotetext{
${ }^{1}$ Alternative Abgrenzungen von „Krisenbetrieben“ auch mit neueren Daten haben quantitativ ähnliche Ergebnisse erbracht (Bellmann und Gerner 2011a, 2011b).

${ }^{2}$ Der erste Wert und die Differenz sind signifikant.
}

sonders groß war. Der Sachverständigenrat zur Begutachtung der gesamtwirtschaftlichen Entwicklung (2010) sowie Burda und Hunt (2011) haben den verhältnismäßig geringen Anstieg der Bruttolöhne und -gehälter vor (!) der Krise betont. Damit ergaben sich zumindest günstige Voraussetzungen für die Weiterbeschäftigung. Dazu gehörte auch die Verknappung des Arbeitsangebots durch gesunkene Schulabgängerzahlen, die Nutzung von Arbeitszeitkontensystemen, die sich wie bereits erwähnt in der letzten Dekade boomartig entwickelt hat, die Rolle der betrieblichen Bündnisse für Beschäftigungs- und Standortsicherung und die Kurzarbeiterregelungen, als wichtigen Beitrag der Bundesagentur für Arbeit. Die drei zuletzt genannten Institutionen standen im Mittelpunkt der Studien auf der Basis des IAB-Betriebspanels. Analysen zum Beschäftigungseffekt der Kurzarbeiterregelung haben auf der Basis der Daten des IAB-Betriebspanels Bellmann und Gerner (2011a), Boeri und Brücker (2011) und Bellmann et al. (2012) vorgelegt. In diesen Studien wurde ein das betriebliche Beschäftigungsniveau stabilisierender Effekt der Kurzarbeiterregelung ermittelt, wobei unterschiedliche methodische Ansätze verwendet wurden.

Unser besonderes Interesse galt aber der Rolle der betrieblichen Bündnisse für Beschäftigungs- und Standortsicherung, um die es eigentlich seit einigen Auseinandersetzungen um die Vereinbarungen im Jahre 2004 z.B. bei Siemens und Daimler Chrysler und in 2005 bei Continental ruhiger geworden war. Ähnlich wie Öffnungsklauseln haben sie sich im Zuge der tarifpolitischen Dezentralisierung seit Mitte der 1990er Jahre im deutschen System der industriellen Beziehungen etabliert - darauf wurde bereits eingegangen. Im IAB-Betriebspanel wurde erstmals 2006 ausführlich nach der Existenz, der Einführung, der Dauer, den Zielen und der Art der Vereinbarungen gefragt (Ellguth und Kohaut 2008). In den folgenden Wellen wurden das Thema von einigen Ausnahmen abgesehen, immer wieder aufgegriffen, so dass Längsschnittauswertungen auch bereits vor der globalen Krise 2008/09 durchgeführt werden konnten (Bellmann et al. 2008).

Insgesamt sind die Ergebnisse der bislang vorliegenden empirischen Studien über die betrieblichen Bündnisse und ihre Beschäftigungswirkungen als enttäuschend für die Akteure zu betrachten (Hübler 2005; Bellmann et al. 2008). Wegen ihrer Schwere bot die 2008/2009er Krise die besondere Gelegenheit die Effekte von Bündnissen auf die Beschäftigungsentwicklung noch einmal zu untersuchen. $\mathrm{Zu}$ mal sie geradezu als Mustersituation zu betrachten ist, die ein hohes Maß an Flexibilität und die Bereitschaft der Sozialpartner, ,nachzuverhandeln“ dringend erforderlich machte, um Arbeitsplatzverluste zu vermelden und die betriebliche Wettbewerbsfähigkeit zu erhalten. Weiterhin kann der Abschluss und die Existenz betrieblicher Bündnisse für Beschäftigungs- und Standortsicherung sowohl als Signal 
Tab. 2 Beschäftigungsentwicklung in Betrieben mit betrieblichem Bündnis für Beschäftigung

\begin{tabular}{llllllll}
\hline & \multicolumn{2}{l}{ Krisenbetriebe } & & & \multicolumn{2}{l}{ Nicht-Krisenbetriebe } \\
\cline { 2 - 3 } & Mit Bündnis & Ohne Bündnis & Differenz & & Mit Bündnis & Ohne Bündnis & Differenz \\
\hline $2007 / 2006$ & 0,026 & 0,014 & 0,012 & 0,025 & 0,006 & 0,019 \\
$2008 / 2007$ & 0,043 & 0,016 & 0,027 & 0,037 & $-0,004$ & 0,041 \\
$2009 / 2008$ & $-0,015$ & $-0,070^{*}$ & $0,055^{*}$ & & 0,000 & $-0,014$ & 0,014 \\
\hline
\end{tabular}

* signifikant auf dem $10 \%$-Niveau

Quelle: Eigene Berechnungen auf der Grundlage des IAB-Betriebspanels 2008-2009 unter Verwendung einer Reihe von weiteren Variablen, Anzahl der Betriebe: 9196

wie auch als Institutionalisierung für diese Bereitschaft der Sozialpartner angesehen werden.

Die Ergebnisse der ökonometrischen Analysen von Bellmann und Gerner (2011a, 2011b) zeigen, dass der Anteil der Betriebe, die ein betriebliches Bündnis abgeschlossen haben, innerhalb der Gruppe der Betriebe, die von der Krise 2008/09 betroffen waren, etwa bei $3 \%$ lag und sich kaum verändert hat. Der Anteil der dort Beschäftigten reduziert sich allerdings, wenn auch nur geringfügig von 19,1\% (2006) auf 18,4\% (2009). Der Anteil der nicht von der Krise betroffenen Betriebe mit einem betrieblichen Bündnis für Beschäftigungs- und Standortsicherung stieg dagegen von $0,5 \%$ (2006) auf $1,7 \%$ (2009) leicht an. In einem weiteren Schritt wurde der von der Vereinbarung betrieblicher Bündnisse für Beschäftigung ausgehende Beschäftigungseffekt untersucht. Die in der Tab. 2 dargestellten Ergebnisse zeigen, dass sich die Beschäftigung in jedem der betrachteten Jahre, sowohl für Krisenbetriebe als auch für Nicht-Krisenbetriebe in den Betrieben, in denen ein betriebliches Bündnis für Beschäftigung abgeschlossen wurde, günstiger als in Betrieben ohne ein derartiges betriebliches Bündnis entwickelt hat. Besonders interessant ist der deutliche Unterschied in der Beschäftigungsentwicklung im Krisenjahr 2009: Die von der Wirtschafts- und Finanzkrise 2008/2009 betroffenen Betriebe reduzierten die Beschäftigung um 1,5\% (von 2008 auf 2009), wenn in ihrem Betrieb ein betrieblichen Bündnis existierte, während in den Betrieben ohne ein derartiges Bündnis die Beschäftigung um 7,0 \% zurückging. Die Differenz ist auf einem Niveau von $10 \%$ signifikant. Damit zeigt sich, dass sich der Abschluss von betrieblichen Bündnissen für Beschäftigungsund Standortsicherung in der Wirtschafts- und Finanzkrise 2008/2009 positiv auf die Beschäftigungsentwicklung in den betreffenden Betrieben ausgewirkt hat. Da sich der Anteil der Betriebe, die ein betriebliches Bündnis abgeschlossen haben, im Beobachtungszeitraum kaum verändert hat, ist dieser positive Effekt sicherlich auch auf bestimmte Einzelmaßnahmen zurückzuführen, die bereits vor der Krise vereinbart worden sind, und auf das Vertrauen der Sozialpartner, auftretende ökonomische Probleme gemeinsam zu lösen.
Hinzu kommt aber auch eine wesentlich aktivere Rolle der Betriebe, die ein betriebliches Bündnis für Beschäftigung abgeschlossen haben, bei der Durchführung von personalpolitischen Anpassungsmaßnahmen in der Krise 2008/2009, nach denen in der Erhebung 2010 gefragt wurde. Signifikante Unterschiede konnten wir in den Krisenbetrieben beim Abbau von Überstunden bzw. beim Aufbau von Minusstunden, bei der Kurzarbeit, der eingeschränkten Übernahme von Auszubildenden, der NichtWiederbesetzung freier Stellen und bei betriebsbedingten Kündigungen ermitteln. Unterschiede zwischen Bündnisund Nicht-Bündnisbetrieben waren in der Gruppe der NichtKrisenbetriebe zwar ebenfalls vorhanden, aber wesentlich geringer. Das höhere „Aktivitätsniveau“ in den Bündnisbetrieben ist schließlich als Indikator dafür zu sehen, dass es dort eher gelungen ist, einen optimalen Mix an Maßnahmen zu finden, um weitgehend unbeschadet durch die Krise zu kommen.

Darüber hinaus erfolgten weitere Analysen von Bechmann et al. (2011) zur Inanspruchnahme ausgewählter personalpolitischer Maßnahmen zum Erhalt von Beschäftigung aber auch in Verbindung mit einem Beschäftigungsabbau in Krisenbetrieben. Dabei zeigte eine Betrachtung nach Betriebsgrößenklassen, dass mit steigender Betriebsgröße die Häufigkeit der Inanspruchnahme aller genannten Maßnahmen in den Krisenbetrieben stark ansteigt. Während nur knapp jeder zweite von der Krise betroffene Kleinstbetrieb mindestens eine der genannten personalpolitischen Maßnahmen nutzte, traf dies auf fast alle der größeren Krisenbetriebe zu.

Damit sind kleinere Betriebe auf den ersten Blick weniger aktiv als größere Betriebe. Von Bedeutung ist aber, ob die genannten personalpolitischen Maßnahmen überhaupt in Betrieben aller Größenklassen genutzt werden können. Wichtige Interventionsmöglichkeiten der Betriebe wie Überstundenabbau, Nutzung von Arbeitszeitkonten und Langzeitkonten sind in Kleinstbetrieben generell weniger verbreitet und damit auch weniger einsetzbar. Auch das Instrument der Kurzarbeit passt besser auf mittlere und größere Betriebe (vgl. Fischer et al. 2009). Demnach sind diese 
Abb. 5 Inanspruchnahme ausgewählter personalpolitischer Maßnahmen zum Erhalt von Beschäftigung in Krisenbetrieben nach der Betriebsgröße in Deutschland

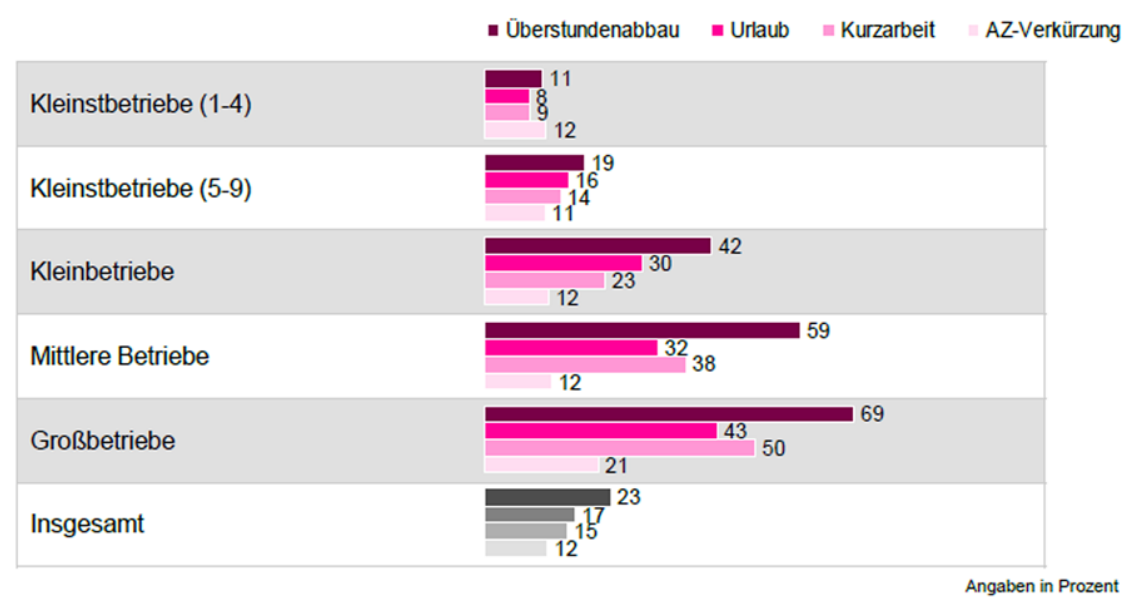

Basis: Alle Betriebe mit negativen Krisenwirkungen

Quelle: IAB-Betriebspanel 2010
Betriebe eher in der Lage, aus den zur Verfügung stehenden personalpolitischen Maßnahmen geeignete Instrumente auszuwählen.

Von den krisenbetroffenen Betrieben wurden jene personalpolitischen Maßnahmen am häufigsten genutzt, die die betriebliche Arbeitszeit reduzierten und gleichzeitig beschäftigungserhaltend wirkten. So erfolgte in fast jedem vierten von der Krise negativ betroffenen Betrieb (23\%) ein gezielter Abbau von Überstunden oder ein Aufbau von Minusstunden auf Arbeitszeitkonten. Auch der angeordnete Urlaub (17\%), Kurzarbeit (15\%) sowie Arbeitszeitverkürzungen außerhalb von Kurzarbeit (12\%) spielten eine Rolle. Mit steigender Betriebsgröße kamen die einzelnen Maßnahmen auch öfter zur Anwendung: Mittlere und größere Krisenbetriebe bedienten sich häufiger bestehender Möglichkeiten, Beschäftigung in den Betrieben zu halten. Insbesondere der gezielte Abbau von Überstunden oder ein Aufbau von Minusstunden auf Arbeitszeitkonten wurden in mittleren und Großbetrieben besonders häufig genannt (vgl. Abb. 5).

Von der Krise negativ betroffene Betriebe nutzten aber nicht nur Maßnahmen zur Anpassung des Arbeitsvolumens an den gesunkenen Arbeitsanfall ohne Reduktion des bestehenden Personals, sondern stellten auch vorgesehene Personalveränderungen zurück. Die Abb. 6 zeigt, dass $17 \%$ der Krisenbetriebe frei werdende Stellen nicht wiederbesetzt haben, $16 \%$ der Krisenbetriebe stellten geplante Personalaufstockungen zurück. Auch diese Maßnahmen wurden von mittleren und größeren Krisenbetrieben häufiger in Anspruch genommen als von Kleinstbetrieben. Dies gilt insbesondere für die Nichtbesetzung frei werdender Stellen, die besonders häufig in mittleren und Großbetrieben erfolgte. Außerdem sprachen Betriebe dieser Größenklassen deutlich häufiger betriebsbedingte Kündigungen aus als Betriebe anderer Größenklassen - wenngleich deutlich seltener als die anderen Maßnahmen.
Mit zunehmender Krisenbetroffenheit wurden ausnahmslos alle personalpolitischen Maßnahmen häufiger von den Betrieben genutzt. Diese Aussage gilt fast uneingeschränkt für die Betriebe aller Größenklassen. Es gibt allerdings Reaktionsmuster der Betriebe, die darauf hindeuten, dass bestimmte Maßnahmen einen besonderen Stellenwert in der Krisenbewältigung hatten. Wenn von der Krise sehr stark betroffene Betriebe ein Instrument deutlich häufiger nutzten als Betriebe, die nur gering krisenbetroffen sind, so spricht dies dafür, dass das jeweilige Instrument den sehr stark von der Krise beeinflussten Betrieben besonders geeignet erschien, der Krise zu begegnen. In Abb. 6 wird deutlich, dass Großbetriebe häufiger alle personalpolitischen Instrumente genutzt haben und bei ihnen die Nichtbesetzung frei werdender Stellen häufiger als betriebsbedingte Kündigungen waren.

In der Abb. 7 zeigt sich die zentrale Bedeutung der Stärke der Betroffenheit von der Krise für die Inanspruchnahme personalpolitischer Maßnahmen ${ }^{3}$ : In von der Krise sehr stark betroffenen Betrieben war der Erhalt von Beschäftigung häufig nicht möglich, und es wurden betriebsbedingte Kündigungen ausgesprochen: Während der Anteil der Betriebe mit betriebsbedingten Kündigungen in gering von der Krise betroffenen Betrieben bei nur $6 \%$ lag, erreichte er in von der Krise sehr stark betroffenen Betrieben $31 \%$. An zweiter Stelle steht die Kurzarbeit, die in gering krisenbetroffenen Betrieben von $5 \%$ der Betriebe genutzt wurde, in sehr stark krisenbetroffenen Betrieben von $27 \%$. Es folgen

\footnotetext{
${ }^{3}$ Als Kriterium gilt der vorhandene Unterschied in der Inanspruchnahme von Maßnahmen in Betrieben mit geringem und in Betrieben mit sehr starkem Kriseneinfluss: Je größer der Unterschied, desto häufiger erfolgt eine Nutzung des jeweiligen Instruments in sehr stark von der Krise betroffenen Betrieben im Vergleich zu Betrieben mit geringem Kriseneinfluss und desto geeigneter erschien es den Betrieben, der Krise damit zu begegnen.
} 
Abb. 6 Inanspruchnahme ausgewählter personalpolitischer Maßnahmen (verbunden mit

Beschäftigungsabbau) in

Krisenbetrieben nach der

Betriebsgröße in Deutschland

\begin{abstract}
Abb. 7 Inanspruchnahme personalpolitischer Maßnahmen in sehr stark und gering von der Krise betroffenen Betrieben in Deutschland
\end{abstract}

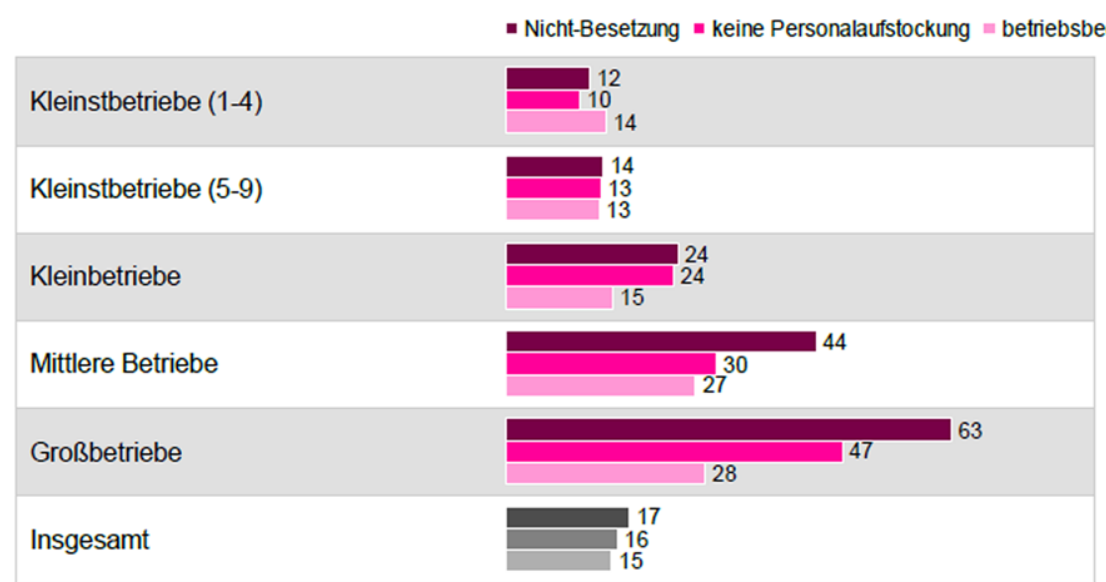

Angaben in Prozent

Basis: Alle Betriebe mit negativen Krisenwirkungen Quelle: IAB-Betriebspanel 2010

\begin{tabular}{|c|c|c|}
\hline Betriebsbedingte Kündigungen & $\begin{array}{lll}6 & \end{array}$ & \multirow{12}{*}{$\begin{array}{l}\text { sehr stark } \\
\text { n gering }\end{array}$} \\
\hline Kurzarbeit & 5 & \\
\hline Nichtbesetzung freier Stellen & $\begin{array}{l}\square \\
\end{array}$ & \\
\hline Abbau Überstunden & $\begin{array}{ll} & 25\end{array}$ & \\
\hline Angeordneter Urlaub & 422 & \\
\hline Kein Personalaufbau & 621 & \\
\hline Verkürzung der Arbeitszeit & 515 & \\
\hline Abbau Befristung & 12 & \\
\hline Verstärkte Weiterbildung & $\square^{6}$ & \\
\hline Geringere Übernahme von Azubi & $\sqrt{2} 6$ & \\
\hline Abbau Leiharbeit & $\pi^{4}$ & \\
\hline Sonstiges & 4 & \\
\hline
\end{tabular}

Angaben in Prozent

Ale Betriebe mit negativen Krisenwirkungen

Quelle: IAB-Betriebspanel 2010

der angeordnete Urlaub (4\% vs. $22 \%$ ) sowie Nichtbesetzung frei werdender Stellen (9 \% vs. $26 \%$ ). Bei den anderen personalpolitischen Maßnahmen sind die Unterschiede in der Nutzung deutlich geringer, werden zwar ebenfalls am häufigsten von sehr stark krisenbetroffenen Betrieben genutzt, aber die Unterschiede in der Nutzung sind deutlich geringer.

Dieser Bericht über die Forschungen zur globalen Krise 2008/09 wäre unvollständig, wenn ich nicht den Hinweis der OECD (2012) aufgenommen hätte, dass die betrieblichen Reaktionen in der Krise entscheidend auch für die makroökonomischen Arbeitsmarktwirkungen waren. Die Rolle der nationalen Institutionen (Kündigungsschutz, Regelungen für atypische Beschäftigungsverhältnisse, Kurzarbeit und Arbeitszeit) für die bereits erwähnten Unterschiede in der Arbeitsmarktperformance verschiedener Länder ist da- mit offensichtlich. Hinzu kommt die Bedeutung der an den Lohnverhandlungen beteiligten Institutionen:

"Co-ordinated wage-bargaining institutions can contribute to both good structural performance and labour market resilience. More specifically, co-ordination appears to be important in achieving low structural unemployment rates and in mitigating the direct impact of shocks on employment by facilitating adjustments to wages and/or working-time“" (OECD 2012).

\section{Schlussbemerkungen}

In diesem Beitrag wurden die wichtigsten Erfolgsfaktoren des IAB-Betriebspanels im einzelnen diskutiert dazu gehört die Verfügbarkeit der Betriebsdatei(en) der Beschäf- 
tigtenstatistik der BA als hervorragende Ziehungsgrundlage der Stichprobe ebenso wie die sich daraus ergebende Möglichkeit der Verknüpfung der Daten des IAB-Betriebspanels mit den administrativen Daten der Beschäftigtenstatistik der BA, dem hohen Rücklauf von über $85 \%$ bei den wiederholt befragten Betrieben und die exzellente Qualität der erhobenen Daten als Voraussetzung für methodisch und inhaltlich anspruchsvolle Panelanalysen. Hinzu kommen die exzellenten Möglichkeiten des Datenzugangs für die Scientific Community über das FDZ und die dynamische Entwicklung des Fragebogens und die Berücksichtigung neuer Fragen im IAB-Betriebspanel. Diese hervorragenden Bedingungen führten dazu, dass es Wissenschaftlern aus dem IAB und externen Wissenschaftlern gelungen ist, eine Vielzahl von Studien zu erstellen sowie in nationalen und internationalen Journals zu veröffentlichen. Der institutionelle Aufbau und die Organisation des IAB-Betriebspanels haben die erforderlichen Anpassungen und Weiterentwicklungen ermöglicht. Diesen Aspekten widmet sich der vorliegende Beitrag.

Die im Zentrum der wissenschaftlichen Untersuchungen stehenden Themenbereiche sind die

(1) betriebliche Flexibilität

(2) Fachkräftebedarf, betriebliche Aus- und Weiterbildung

(3) Innovationen sowie technischer und organisatorischer Wandel

(4) Industrielle Beziehungen sowie

(5) die Auswirkungen der Finanz- und Wirtschaftskrise 2008/2009 auf den Arbeitsmarkt.

Bevor auf die entsprechenden Fragestellungen und ihre Weiterentwicklung im einzelnen eingegangen wird, muss zumindest erwähnt werden, dass v.a. externe Wissenschaftler andere, hier nicht aufgeführte Fragen des IAB-Betriebspanels ausgewertet und entsprechende Veröffentlichungen vorgelegt haben.

(1) Durch die Globalisierung und den zunehmenden Wettbewerb auf den Beschäftigungs- und Absatzmärkten steigt die Notwendigkeit für die Betriebe, sich an diese Entwicklungen flexibel anzupassen. Bei der internen Flexibilität stand zu Beginn der Erhebung des IAB-Betriebspanels die Nutzung vorsetzten Arbeitszeiten, sowie von Überstunden und später Arbeitszeitkonten im Mittelpunkt. Bei externer Flexibilität wurde die Rolle von Einstellungen und Entlassungen und die zunehmende Bedeutung erlangenden atypischen Beschäftigungsformen (wie Befristungen, Leiharbeit, geringfügige Beschäftigung, Praktika und Werkverträge) untersucht. Wichtige Erweiterungen wurden durch die Abfrage von Gründen für die Nutzung der diversen atypischen Beschäftigungsformen sowie die Übernahme und den Verbleib von befristet Beschäftigten vorgenommen.

(2) Nicht zuletzt hat das IAB-Betriebspanel 2006 die herausragende Bedeutung der Qualität des Fachkräfteangebots als Standortfaktor gezeigt. Die Fragen zu den Neueinstellungen von Fachkräften, die unmittelbare Suche nach Fachkräften und die unbesetzten Stellen für Fachkräfte wurden ergänzt durch Fragen nach dem zu erwartenden Personalbedarf, der über Neueinstellungen gedeckt werden soll, aber auch durch Stellenbesetzungen über interne Wechsel, nach Schwierigkeiten bei der Besetzung von Fachkräftestellen, den dabei eingegangenen Kompromissen und den personalpolitischen Maßnahmen zur Deckung des zukünftigen Fachkräftebedarfs. Weiterhin wurden regelmäßig Fragen zur betrieblichen Aus- und Weiterbildung aufgenommen. Dazu gehören Fragen nicht nur zur Berechtigung und Teilnahme an der betrieblichen Berufsausbildung, sondern auch zur Weiterbeschäftigung von Ausbildungsabsolventen im Ausbildungsbetrieb. Im Rahmen des IAB-Betriebspanels werden die betrieblichen Weiterbildungsaktivitäten seit 2007 jährlich erhoben; zuvor gab es einen zweijährigen Rhythmus. Im IAB-Betriebspanel werden Informationen zur Teilnahme an beruflicher Weiterbildung in Betrieben, die von diesen veranlasst oder zumindest teilweise finanziert wird, erhoben. Hinzu kommen Angaben für die Form der Weiterbildung (externe und interne Kurse, Weiterbildung am Arbeitsplatz, Arbeitsplatzwechsel, selbst gesteuertes Lernen, Qualitätszirkel u.a.), die Teilnahme von Beschäftigten differenziert nach Qualifikationen, Geschlecht und Alter.

(3) Innovationen, technischer und organisatorischer Wandel lösen oftmals einen zusätzlichen Qualifikationsbedarf aus. Im IAB-Betriebspanel werden deshalb drei Typen von Produktinnovationen (Weiterentwicklungen, Imitationen und Marktneuheiten), Prozessinnovationen, der technische Stand der Maschinen und Anlagen sowie Änderungen der betrieblichen Aufgaben- und Produktionsstruktur erfasst. Im Mittelpunkt der Auswertungen stand die Analyse der Inzidenz, aber auch die Ermittlung von Lohn- und Beschäftigungseffekten. Interessanterweise sprechen die erhaltenen empirischen Ergebnisse nicht eindeutig für einen skill-bias des organisatorischen Wandels, durchaus im Einklang mit Studien für andere Länder.

(4) Die Erosion der Flächentarifverträge und die Stärkung der betrieblichen Aushandlungsebene haben in den letzten 20 Jahren besonders große Aufmerksamkeit gefunden. Im IAB-Betriebspanel sind aber nicht nur Fragen zur Bindung von Branchen- und Firmentarifverträgen, übertariflichen Entlohnung, der Orientierung von Tarifverträgen, sondern auch zur Bedeutung und dem Inhalt von tariflichen Öffnungsklauseln sowie zum Abschluss von betrieblichen Bündnissen für Beschäftigungs- und Standortsicherung enthalten: Nach der Existenz eines Betriebs- oder Personalrats wird in jeder Welle des IAB-Betriebspanels gefragt, gelegentlich ergänzt durch die Abfrage der Existenz anderer Mitbestimmungsorgane.

(5) Der beim Rückgang des Bruttoinlandsprodukts in Deutschland um fast $6 \%$,milde“ Beschäftigungseffekt der 
Wirtschafts- und Finanzkrise 2008/2009 ist aus internationaler Perspektive erstaunlich. Diese Krise hat die Bedeutung von Arbeitsmarktinstitutionen unterstrichen sowie die Gewerkschaften und die Mitbestimmung in einem positiveren Licht erscheinen lassen. Mit Analysen auf der Basis des IAB-Betriebspanels konnten wir zeigen, dass die Konzentration der Krise auf wenige exportintensive Wirtschaftszweige, die starke Zunahme der Nutzung von Arbeitszeitkonten im Zeitraum 1999 bis 2007, die Inanspruchnahme des Kurzarbeitergelds sowie die Wirkung von betrieblichen Bündnissen für Beschäftigungs- und Standortsicherung von entscheidender Bedeutung für die erfolgreiche Bewältigung der globalen Rezession waren. Weitergehende Untersuchungen galten der Nutzung im beschäftigungssichernden personalpolitischen Maßnahmen und den Formen des Personalabbaus in den von der Wirtschafts- und Finanzkrise besonders betroffenen Betrieben.

Natürlich ist es schwierig, die zukünftig für die Wissenschaft und die an beschäftigungspolitischen Fragen interessierte Öffentlichkeit wichtigen Themen zu bestimmen. Aus aktueller Sicht spricht vieles dafür, dass die Globalisierung die Ursache häufiger und heftigerer Krisen sein wird (Roubini 2010), so dass mikroökonometrische Analysen zu den Wirkungen der auftretenden Krisen weiterhin einen besonderen Stellenwert haben sollten. Dies gilt auch für die vertiefenden Untersuchungen der Auswirkungen der betrieblichen Aus- und Weiterbildung, der Einstellung und Beschäftigung Älterer, der Beteiligung der Mitarbeiter, der Nutzung von Langzeitkonten und der Frauen in Führungspositionen.

Weiterhin werden wichtige Projekte in Angriff genommen wie die Prüfung einer Umstellung der Befragungsmethode auf computergestützte Erhebungsverfahren, die experimentelle Validierung von Indikatoren des Managerverhaltens in Betriebsbefragungen, die Durchführung einer Firmenbefragung zu den wirtschaftlichen Aktivitäten seit der EU-Osterweiterung (Münich et al. 2012; Moritz und Hecht 2013; Pflüger et al. 2013; Hecht et al. 2013). Von zentraler Bedeutung ist die Verknüpfung der Daten des IABBetriebspanels mit administrativen Daten v.a. der Beschäftigtenstatistik der BA sowie die Durchführung von zusätzlichen Erhebungen bei Betrieben und Beschäftigten gemeinsam mit dem FDZ und externen Partnern, wie der Universität Köln und des ZEW Mannheim, im BMAS-Projekt „Arbeitsqualität und wirtschaftlicher Erfolg“".

\section{Kurzfassung}

Die Ziele dieses Beitrags bestehen darin, die methodischen Vorteile des IAB-Betriebspanels dazustellen und einen Überblick über die wichtigsten Studien auf der Basis dieses Datensatzes und des LIAB zu geben. Der LIAB ist der Linked-Employer-Employee-Datensatz des IAB bestehend aus dem IAB-Betriebspanel und den Daten der Beschäftigtenstatistik der Bundesagentur für Arbeit.

Als Schlüssel für den Erfolg des IAB-Betriebspanels, das 1993 gestartet wurde, sind folgende Faktoren zu nennen: (1) Der hohe Rücklauf von mehr als $85 \%$ bei den wiederholt befragten Betrieben, (2) die für Panelanalysen erforderliche exzellente Datenqualität, (3) die einzigartige Möglichkeit der Nutzung des Betriebsregisters der Beschäftigtenstatistik der Bundesagentur für Arbeit, nicht nur für die Ziehung der Stichproben, sondern auch um Linked-EmployerEmployee-Datensätze aufzubauen und (4) der Datenzugang für externe Wissenschaftler über das Forschungsdatenzentrum des IAB.

Ebenso wichtig für den Erfolg ist die dynamische Entwicklung und die Berücksichtigung neuer Fragen im 24 Seiten umfassenden Fragebogen des IAB-Betriebspanels. Die fünf wichtigsten Themenbereiche sind (1) die betriebliche Flexibilität, (2) die Nachfrage nach Fachkräften sowie die betriebliche Aus- und Weiterbildung, (3) Innovationen sowie der technische und organisatorische Wandel, (4) die industriellen Beziehungen sowie (5) die Auswirkungen der Finanz- und Wirtschaftskrise 2008 auf den Arbeitsmarkt.

(1) Die Globalisierung und der zunehmende Wettbewerb zwingen die Betriebe, sich an die Markterfordernisse anzupassen. Die Verbreitung und die Effekte der Maßnahmen der internen Flexibilität, wie z.B. der Überstunden, Arbeitszeitkonten und Schichtarbeit werden ebenso untersucht wie die Maßnahmen der externen Flexibilität, deren Bedeutung in den letzten Jahren gemessen an ihren Beschäftigtenanteilen gewachsen ist. Insbesondere Informationen über verschiedene atypische Beschäftigungsformen wie befristete Beschäftigung, Zeitarbeit, Teilzeitarbeit und Praktika sind verfügbar. Der Übergang von atypische in permanente Beschäftigungsverhältnisse kann ebenfalls untersucht werden.

(2) Das IAB-Betriebspanel 2006 zeigte die überragende Bedeutung der Qualität des Fachkräfteangebots als betrieblichen Standortfaktor für die Betriebe. Es werden regelmäBig verschiedene Indikatoren zur Ermittlung des Fachkräftebedarfs wie z.B. die Suche nach Fachkräften, die Besetzung von Fachkraftstellen und die Herausforderung bei der Rekrutierung von Arbeitskräften erhoben. Darüber hinaus werden die Betriebe seit einigen Jahren nach den von ihnen eingegangen Kompromissen bei der Stellenbesetzung befragt. Weitere Fragen beziehen sich auf die betriebliche Aus- und Weiterbildung. In diesem Zusammenhang haben Studien über die Weiterbildungsteilnahme von Männern und Frauen sowie verschiedener Qualifikations- und Altersgruppen große Aufmerksamkeit in der Fachöffentlichkeit erlangt. Dies trifft auch zu für die empirische Evidenz zu den Hindernissen bei der Einstellung älterer Beschäftigter und den vergleichsweise geringen betrieblichen Engagement bei Maßnahmen der altersspezifischen Personalpolitik.

(3) Sowohl Produkt- und Prozessinnovationen als auch der technologische und organisatorische Wandel führen $\mathrm{zu}$ 
einer größeren Nachfrage nach qualifizierten, insbesondere hochqualifizierten Beschäftigten. Jedoch zeigen Studien, dass der durch Dezentralisierung von Kompetenz und Verantwortung, die Einführung von Gruppenarbeit, Weiterbildung, Anreizentlohnung und flexible Arbeitszeiten und verbesserte Qualitätssicherung gemessene organisatorische Wandel keine eindeutig destabilisierenden Beschäftigungseffekte für Arbeitskräfte ohne formalen Berufsabschluss hat.

Die Analyse der regionalen Determinanten verschiedener Innovationsaktivitäten im Rahmen eines Drei-EbenenModells zeigt, dass Prozess- und radikale Produktinnovationen signifikant durch die Arbeitslosenquote und den Anteil von Absolventen in den Fächern Mathematik, Ingenieurwissenschaften, Naturwissenschaften und Technik sowie der Nähe zu Forschungs- und Technologiezentren sowie Hochschulen beeinflusst werden.

(4) Die Erosion des Flächentarifvertrages in Deutschland, die durch das IAB-Betriebspanel dokumentiert wird, hat einen großen Einfluss auf verschiedene Felder der Arbeitsmarktforschung: Während des Zeitraums 1996 bis 2011 hat der Anteil der Beschäftigten, die in Betrieben arbeiten, die an Branchentarifverträge gebunden sind, in Westdeutschland von $70 \%$ auf $54 \%$ und in Ostdeutschland von $56 \%$ auf $37 \%$ abgenommen. Das bedeutet, dass der für Westdeutschland im Jahre 2011 berechnete Wert niedriger ist als der entsprechende Wert für Ostdeutschland am Anfang des Beobachtungszeitraums. Allerdings hat sich während die Tarifbindung gesunken ist, die Orientierung der Betriebe an Branchentarifverträgen verstärkt.

(5) Die Finanz- und Wirtschaftskrise 2008/2009 hat das Bruttoinlandsprodukt in Deutschland um $6 \%$ reduziert. Die Krise traf besonders die exportierenden Unternehmen. Gründe für die moderate Reaktion des Arbeitsmarktes wurden im Hinblick auf verschiedene Arbeitsmarktinstitutionen in Deutschland untersucht. Die empirische Evidenz auf der Basis des IAB-Betriebspanels zeigt, dass das betriebliche Beschäftigungsniveau durch die (reformierte) Kurzarbeiterregelung sowie durch betriebliche Bündnisse für Beschäftigung und Standortsicherung stabilisiert werden konnte. Weiterhin wurden die in der Krise ergriffenen personalpolitischen Maßnahmen untersucht. Da die verstärkte Globalisierung zu häufigere und intensivere Wirtschaftskrisen in Zukunft führen könnte, wird die Projektgruppe „IABBetriebspanel“ dieses Thema auch weiterhin auf ihre Forschungsagenda setzen. Die betrieblichen Reaktionen sind außerdem sehr relevant für die Diskussion der makroökonomischen Stabilisierungspolitik.

\section{Executive summary}

The objectives of this paper are to present the methodological advantages of the IAB Establishment Panel Survey and to survey of the most relevant scientific contributions of analyses based on this data set as well as the LIAB. The LIAB is a linked-employer-employee which comprises of the IAB Establishment Panel Survey and the employee data from the Employment Statistics of the Federal Employment Agency.

The success of the IAB Establishment Panel which started in 1993 can be ascribed to (1) the high response rate of over $85 \%$ for repeatedly asked establishments, (2) the excellent data quality necessary for panel analyses, (3) the unique possibility to use the establishment register of the Employment Statistics of the Federal Employment Agency not only to draw the samples but also to construct linkedemployer-employee data sets, and (4) external researchers' data access via the IAB Research Data Centre.

Both, the dynamic development of the questionnaire and the consideration of new questions in the 24 pages-long questionnaire were similarly important for the success of the IAB Establishment Panel. The five most relevant research topics in the questionnaire are: (1) the establishments' flexibility, (2) the demand of skilled personnel, vocational and further training (3) innovations as well as technological and organizational change, (4) industrial relations, and (5) the labor market effects of the Great Recession 2008/2009.

(1) The globalization and the increasing competition forces the establishments to adjust to the needs of the markets. The incidence and effects measures of internal flexibility e.g. over-time work, working-time accounts and shift work are investigated as well as the measures of external flexibility, which gained larger proportions in the last years. Especially, information about different forms of atypical employment like fixed-term contracts, temporary jobs, parttime work and internships are available. The transition from atypical into permanent jobs can also be considered.

(2) The IAB Establishment Survey 2006 demonstrated the overwhelming importance of the employment of skilled personnel for the establishments. Questions concerning the recruitment of employees but also about the challenges companies faced with are asked regularly. As an innovation we raised questions concerning compromises of establishments which seek to fill their vacancies. Furthermore, studies about firms' initial vocational training and further training are conducted. In this context investigations about the participation of men and women as well as of members of different qualification and age groups attract high public interest. This is also true for the empirical evidence about the hiring difficulties of older employees and their low participation rates in measures intended to improve their employability.

(3) Product and process innovations as well as technological and organizational change lead to an increased demand for qualified labor, especially higher educated. However, studies demonstrate that organizational change characterized by measures fostering delayering, teamwork, job ro- 
tation, further training, incentive payment and flexible working time, relocation of responsibilities and product quality all do not show clearly destabilizing effects on employment for low skilled workers.

Analyzing the regional determinants of different innovation types using three-level random effect logit models it is revealed that process and radical innovations are significantly affected by the unemployment rate and the share of graduates in mathematics, informatics, natural sciences and technological sciences.

(4) The erosion of sectoral bargaining coverage in Germany documented by the IAB Establishment Panel seems to exert a large impact on various fields of labour market research: During the years 1996-2011 the proportion of employees working in establishments covered by sectoral bargaining agreements decreased from $70 \%$ to $54 \%$ in West Germany and from $56 \%$ to $37 \%$ in East Germany. Thus, the value calculated for West Germany in 2011 reached a lower level than the respective value for East Germany at the beginning of the observation period 1996. However, at the same time as bargaining coverage declined the establishments' orientation towards the standards set by bargaining agreement increases.

(5) The Great Recession 2008/09 decreased the Gross Domestic Product by $6 \%$ in Germany. The crisis especially hit exporting companies. Reasons for the resilience of the labor market were investigated by the consideration of different German labor market institutions. Empirical evidence based on the IAB Establishment Panel Survey demonstrates the employment stabilizing effects of the (modernized) short-time regulation and company-level pacts for employment and competitiveness. Further studies considered the adoption of personnel measures during the crisis. As the ongoing process of globalization might cause more frequent and intensive crises in future, the project group "IAB Establishment Panel" will take up this topic for further research. The firms' reactions are also of high relevance for the discussion of the macroeconomic stabilization policies.

\section{Literatur}

Addison, J.T., Bellmann, L., Schnabel, C., Wagner, J.: The reform of the German works constitution act. A critical assessment. Ind. Relat. 43(2), 392-420 (2004)

Addison, J.T., Bellmann, L., Schank, T., Teixera, P.: The demand for labor: an analysis using matched employer-employee data from the German LIAB. Will the high unskilled worker own-wage elasticity please stand up? J. Labor Res. 29(2), 114-137 (2008)

Alda, H., Bellmann, L.: Organisatorische Änderungen und betriebliche Beschäftigungs- und Qualifikationseffekte 1999-2001. Mitt. Arb.markt- Berufsforsch. 35(4), 523-545 (2002)

Alda, H., Bender, S., Gartner, H.: The linked employer-employee dataset created from the IAB establishment panel and the processproduced data of the IAB (LIAB). Schmollers Jahrb. Wirtsch.Soz.wiss. 125(2), 327-336 (2005)
Allmendinger, J., Kohlmann, A.: Datenverfügbarkeit und Datenzugang am Forschungsdatenzentrum der Bundesagentur für Arbeit im Institut für Arbeitsmarkt- und Berufsforschung. Allg. Stat. Arch. 89(2), 159-182 (2005)

Atkinson, J.: Flexibility, uncertainty and manpower management, IMSreport No. 89. London (1985)

Bauer, T.K., Bender, S.: Flexible work systems and the structure of wages. Evidence from matched employer-employee data. IZA discussion paper 353 (2001)

Bauer, T.K., Bender, S.: Technological change, organizational change, and job turnover. Labour Econ. 11(3), 265-291 (2004)

Bechmann, S., Dahms, V., Fischer, A., Frei, M., Leber, U., Möller, I.: Beschäftigung, Arbeit und Unternehmertum in deutschen Kleinbetrieben. IAB-Forschungsbericht 7/2011 (2011)

Bechmann, S., Dahms, V., Tschersich, N., Frei, M., Leber, U., Schwengler, B.: Fachkräfte und unbesetzte Stellen in einer alternden Gesellschaft - Problemlagen und betriebliche Reaktionen. IAB-Forschungsbericht 13/2012 (2012)

Beckmann, M., Bellmann, L.: Betriebliche Suche nach qualifizierten Arbeitskräften in West- und Ostdeutschland. In: Backes-Gellner, U., Kräkel, M., Schauenberg, B., Steiner, G. (Hrsg.) Flexibilisierungstendenzen in der Betrieblichen Personalpolitik. Anreize, Arbeitszeiten und Qualifikationen. Beiträge zum 3. Köln-Bonner Kolloquium zur Personalökonomie. Beiträge zur Personal- und Organisationsökonomik, Bd. 7, S. 205-232 (2000). München

Bellmann, L.: Das Betriebspanel des IAB. In: Hujer, R., Rendtel, U., Wagner, G. (Hrsg.) Wirtschafts- und sozialwissenschaftliche Panelstudien: Datenstrukturen und Analyseverfahren. Allg. Stat. Arch. (Sonderheft) 169-182 (1997)

Bellmann, L.: Das IAB-Betriebspanel. Konzeption und Anwendungsbereiche. Allg. Stat. Arch. 86(2), 177-188 (2002)

Bellmann, L.: Datenlage und Interpretation der Weiterbildung in Deutschland. Herausgegeben von der Kommission „Finanzierung Lebenslangen Lernens“. Bielefeld (2003)

Bellmann, L.: Zur Entwicklung der Leiharbeit in Deutschland. Theoretische Überlegungen und empirische Ergebnisse aus dem IABBetriebspanel. Soz. Fortschr. 53(6), 135-142 (2004)

Bellmann, L., Allaart, P.: Reasons for part-time work. An empirical analysis for Germany and the Netherlands. Int. J. Manpow. 28(7), 557-570 (2007)

Bellmann, L., Ellguth, P.: Verbreitung von Betriebsräten und ihr Einfluss auf die betriebliche Weiterbildung. Jahrb. Natl.ökon. Stat. 226(5), 487-504 (2006)

Bellmann, L., Gerner, H.D.: Reversed roles? Wage and employment effects of the current crisis. Res. Labor Econ. 32, 181-206 (2011a)

Bellmann, L., Gerner, H.D.: Company-level pacts for employment in the global crisis 2008/2009: first evidence from representative German establishment-level panel data. Int. J. Hum. Resour. Manag. 23, 3375-3396 (2011b)

Bellmann, L., Gewiese, T.: Die Dynamik der Nutzung von Arbeitszeitkonten. Theoretische Ansätze und Längsschnittanalysen mit dem IAB-Betriebspanel. In: Bellmann, L., Schnabel, C. (Hrsg.) Betriebliche Arbeitszeitpolitik im Wandel. Beiträge zur Arbeitsmarkt- und Berufsforschung, Bd. 288, S. 139-184 (2004). Nürnberg

Bellmann, L., Hartung, S.: Übernahmemöglichkeiten im Ausbildungsbetrieb. In: Eine Analyse mit dem IAB-Betriebspanel. Sozialer Fortschritt, Bd. 59(6/7), S. 160-167 (2010)

Bellmann, L., Janik, F.: Betriebe und Frühverrentung: Angebote, die man nicht ablehnt. Z. Arb.markt Forsch. 42(4), 311-324 (2009)

Bellmann, L., Leber, U.: Weiterbildung für Ältere in KMU. Soz. Fortschr. 57(2), 43-48 (2008)

Bellmann, L., Pahnke, A.: Auswirkungen organisatorischen Wandels auf die betriebliche Arbeitsnachfrage. Z. Arb.markt Forsch. 39(2), 201-233 (2006) 
Bellmann, L., Schank, T.: Innovations, wages and the demand for heterogeneous labour: new evidence from a matched employeremployee data-set. IZA-discussionpaper No. 112 (2000)

Bellmann, L., Stegmaier, J.: Perspektiven der Erwerbsarbeit. Facharbeit in Deutschland. Dokumentation der Expertengespräche. Gesprächskreis Arbeit und Qualifizierung. Friedrich-Ebert-Stiftung, Bonn (2010)

Bellmann, L., Stegmaier, J.: Einfacharbeit in der Krise? Arbeit 20(3), 188-205 (2011)

Bellmann, L., Dörfer, G., Dostal, W., Kühl, J., Lahner, M., Schnur, P., Ulrich, E., Wolfsteiner, M.: Das IAB-Betriebspanel. Ansatz und Aufbau. Mitt. Arb.markt- Berufsforsch. 24(3), 514-530 (1991)

Bellmann, L., Düll, H., Kühl, J., Lahner, M., Lehmann, U.: Flexibilität von Betrieben in Deutschland. Ergebnisse des IABBetriebspanels 1993-1995. Beitrag zum OECD-Projekt „Flexible Enterprise“. Beiträge zur Arbeitsmarkt- und Berufsforschung, Bd. 200 (1996). Nürnberg

Bellmann, L., Bender, S., Schank, T.: Flexibilität der Qualifikationsstruktur aus betrieblicher Sicht. Substitutionalität oder Komplementarität. Jahrb. Natl.ökon. Stat. 219(1/2), 109-126 (1999)

Bellmann, L., Düll, H., Leber, U.: Zur Entwicklung der betrieblichen Weiterbildungsaktivitäten. In: Reinberg, A. (Hrsg.) Arbeitsmarktrelevante Aspekte der Bildungspolitik. Beiträge zur Arbeitsmarkt- und Berufsforschung, Bd. 248, S. 97-124 (2001)

Bellmann, L., Bender, S., Kölling, A.: Der Linked EmployerEmployee-Datensatz aus IAB-Betriebspanel und Beschäftigtenstatistik der Bundesanstalt für Arbeit. In: Kleinherz, G. (Hrsg.) IAB-Kompendium Arbeitsmarkt- und Berufsforschung. Beiträge zur Arbeitsmarkt- und Berufsforschung, Bd. 250, S. 21-30 (2002a)

Bellmann, L., Caliendo, M., Hujer, R., Radić, D.: Beschäftigungswirkungen technisch-organisatorischen Wandels. Eine mikroökonometrische Analyse mit dem Linked IAB-Panel. Mitt. Arb.marktBerufsforsch. 35(4), 506-522 (2002b)

Bellmann, L., Kistler, E., Wahse, J.: Betriebliche Sicht- und Verhaltensweisen gegenüber älteren Arbeitnehmern. Aus Polit. Zeitgesch. 20, 26-34 (2003)

Bellmann, L., Düll, H.: Der unterschiedliche Zugang zur betrieblichen Weiterbildung nach Qualifikation und Berufsstatus. Eine Analyse auf der Basis des IAB-Betriebspanels 1997 für West- und Ostdeutschland. Mitt. Arb. markt-Berufsforsch. 32(1), 70-84 (1999)

Bellmann, L., Cornelißen, T., Hübler, O., Pahnke, A.: Betriebliche Reorganisation, Entlohnung und Beschäftigungsstabilität. Z. Arb.markt Forsch. 41(2/3), 259-285 (2008)

Bellmann, L., Gerlach, K., Meyer, W.: Company-level pacts for employment. Jahrb. Natl.ökon. Stat. 228(5/6), 533-553 (2008)

Bellmann, L., Fischer, G., Hohendanner, C.: Betriebliche Dynamik und Flexibilität auf dem deutschen Arbeitsmarkt. In: Möller, J., Walwei, U. (Hrsg.) Handbuch Arbeitsmarkt 2009. IAB-Bibliothek, Bd. 314, S. 359-401 (2009). Bielefeld

Bellmann, L., Krekel, E.M., Stegmaier, J.: Aus- und Weiterbildung Komplemente oder Substitute? Zur Bildungsbeteiligung kleinerer und mittlerer Betriebe in Deutschland. Report. Z. Weiterbildungsforsch. 1, 41-54 (2010)

Bellmann, L., Ellguth, P., Gerner, H.-D.: Betriebliche Arbeitszeiten Auswertungen des IAB-Betriebspanels. In: Becker-Stoll, F., et al. (Hrsg.) Expertisen zum Achten Familienbericht „Zeit für die Familie“, S. 397-418. ifo-Institut, München (2012)

Bellmann, L., Crimmann, A., Evers, K., Hujer, R.: Regional effects on establishments ' innovation activities in Germany 2006-2010: A multi-level approach. IZA discussionpaper (im Erscheinen) (2013)

Bender, S., Heining, J.: The research-data centre in research-datacentre approach: a first step towards decentralised international data sharing. FDZ-Methodenreport 07/2011, Nürnberg (2011)

Bender, S., Dieterich, I., Schmucker, A., Seth, S.: FDZ-Jahresbericht 2011/2012, Nürnberg (im Erscheinen) (2013)
Blien, U., von Hauff, M., Horbach, J.: Beschäftigungseffekte von Umwelttechnik und umweltorientierten Dienstleistungen in Deutschland. Mitt. Arb.markt- Berufsforsch. 33(1), 126-135 (2000)

Bockmann, B., Zwick, T.: Betriebliche Determinanten der Beschäftigung älterer Arbeitnehmer. Z. Arb.markt Forsch. 37(1), 53-63 (2004)

Boeri, T., Brücker, H.: Short-time work benefits revisited. Some lessons from the great recession. Econ. Policy 26(68), 697-765 (2011)

Boockmann, B., Hagen, T.: Befristete und andere ,atypische“ Beschäftigungsverhältnisse: Wird der Arbeitsmarkt funktionsfähiger? Z. Arb.markt Forsch. 38(2/3), 305-324 (2005)

Brussig, M., Bellmann, L.: Betriebliche Einflussfaktoren auf Rekrutierungen Älterer und deren Bewerbungen. Z. Pers.forsch. 22(1), 35-57 (2008)

Burda, M., Hunt, J.: What explains the German labor market miracle in the great recession. Brooking Pap. Econ. Act. 42(1), 273-335 (2011)

Card, D., Heining, J., Kline, P.: Workplace heterogeneity and the rise of West German wage inequality. NBER working paper 18522 (2012)

Dietrich, H., Gerner, H.-D.: Betriebliches Ausbildungsverhalten und Geschäftserwartungen. Soz. Fortschr. 57(4), 87-93 (2008)

Dietz, M., Kettner, A., Kubis, A., Leber, U., Müller, A., Stegmaier, J.: Unvollkommene Ausgleichsprozesse am Arbeitsmarkt - Analysen zur Arbeitskräftenachfrage auf Basis des IAB-Betriebspanels und der IAB-Erhebung des Gesamtwirtschaftlichen Stellenangebots. IAB-Forschungsbericht 8/2012 (2012)

Dostal, W., et al.: Das IAB-Betriebspanel - Testphase im Jahr 1992. Ein Sachstandsbericht. Mitt. Arb.markt- Berufsforsch., 26(3), 399-422 (1993)

Düll, H., Bellmann, L.: Betriebliche Weiterbildungsaktivitäten in Westund Ostdeutschland. Eine theoretische und empirische Analyse mit den Daten des IAB-Betriebspanels 1997. Mitt. Arb.markt- Berufsforsch. 31(2), 205-225 (1998)

Düll, H., Ellguth, P.: Atypische Beschäftigung: Arbeit ohne betriebliche Interessenvertretung? Empirische Analysen mit dem IABBetriebspanel zum Einfluß von Betriebsräten auf befristete und geringfügige Beschäftigung. WSI-Mitt. 52(3), 165-176 (1999a)

Düll, H., Ellguth, P.: Betriebliche Strukturen der Teilzeitbeschäftigung in West- und Ostdeutschland. Ergebnisse aus dem IABBetriebspanel 1993, 1996 und 1998. Mitt. Arb.markt- Berufsforsch. 32(3), 269-280 (1999b)

Dustmann, C., Ludsteck, J., Schönberg, U.: Revisiting the German wage structure. Q. J. Econ. 124(2), 843-881 (2009)

Ellguth, P.: Betriebe ohne Betriebsrat. Welche Rolle spielen betriebsspezifische Formen der Mitarbeitervertretung? Ind. Bez. 12(2), 149-176 (2005)

Ellguth, P., Kohaut, S.: Ein Bund fürs Überleben? Betriebliche Vereinbarungen zur Beschäftigungs- und Standortsicherung. Ind. Bez. 15(3), 209-232 (2008)

Ellguth, P., Kohaut, S.: Tarifbindung und betriebliche Interessenvertretung. Aktuelle Ergebnisse aus dem IAB-Betriebspanel 2011. WSI-Mitt. 65(4), 297-305 (2012)

European Commission: Performance of the European Union Labour market: results of an ad hoc labour marktet survey covering employers and employees. European economy. Reports and studies Nr. 3 (1995)

European Foundation for the Improvement of Living and Working Conditions: Restructuring in recession. Dublin (2009)

Fischer, G., Dahms, V., Bechmann, S., Bilger, F., Frei, M., Wahse, J., Möller, I.: Langfristig handeln, Mangel vermeiden: Betriebliche Strategien zur Deckung des Fachkräftebedarfs. Ergebnisse des IAB-Betriebspanels 2007. IAB-Forschungsbericht 3/2008 (2008)

Fischer, G., Janik, F., Müller, D., Schmucker, A.: The IAB establishment panel: things users should know. Schmollers Jahrb. Wirtsch.Soz.wiss. 129(1), 133-148 (2009) 
Gerlach, K., Hübler, O., Giesecke, D., Lorenz, W., Schmidt, E.M., Wagner, J.: Studie zu konzeptionellen und methodischen Aspekten eines Betriebspanels. Institut für Quantitative Wirtschaftsforschung, Hannover (1990)

Gerlach, K., Hübler, O., Meyer, W.: Investitionen, Weiterbildung und betriebliche Reorganisation. Mitt. Arb.markt- Berufsforsch. 35(4), 546-565 (2002)

Gerner, H.-D.: Arbeitszeitverlängerung, Arbeitszeitkonten und Teilzeitbeschäftigung. Ökonometrische Analysen. IAB-Bibliothek, Bd. 322 (2010). Bielefeld

Gerner, H.-D.: Die Produktivitätsentwicklung und die Rolle von Arbeitszeitkonten während der Großen Rezession 2008/2009. Ergebnisse auf der Grundlage des IAB Betriebspanels. Z. Personalforsch. 26(1), 30-47 (2012)

Günther, J., Marek, P.: Einflussfaktoren betrieblicher Innovationstätigkeit: Kleine Betriebe in Ostdeutschland mit viel Potenzial. Wirtsch. Wandel 17(7/8), 276-282 (2011)

Hagen, T.: Does fixed-term contract employment raise firms' adjustment speed? Evidence from an establishment panel for West Germany. Jahrb. Natl.ökon. Stat. 223(4), 403-421 (2003)

Hamermesh, D.: Leeping into the future of labor economics: the research potential of linking employer and employee data. J. Labor Econ. 6, 25-41 (1999)

Hammer, M., Champy, J.: Business Reengineering - die Radikalktur für das Unternehmen (1994). Frankfurt/New York

Hauptmanns, P., Ostendorf, B.: Methodische Erfahrungen und Probleme bei Betriebsbefragungen. Anmerkungen nach drei Wellen des NIFA-Panels im deutschen Maschinenbau. Arbeitspapier Z23/94, Bochum (1994)

Hecht, V., Litzel, V., Scheffler, J.: The ReLOC project-method report for implementing a cross-border company survey in Germany and the Czech Republic. IAB-Forschungsbericht 4/2013 (2013)

Heywood, J.S., Jirjahn, U., Tsertsvadze, G.: Hiring older workers and employing older workers: German evidence. J. Popul. Econ. 23, 595-615 (2010)

Heywood, J.S., Jirjahn, U., Tsertsvadze, G.: Part-time work and the hiring of older workers. Appl. Econ. 43, 4239-4255 (2011)

Hohendanner, C., Bellmann, L.: Interne und externe Flexibilität. WSIMitt. 59(5), 241-246 (2006)

Hohendanner, C., Gerner, H.-D.: Die Übernahme befristet Beschäftigter im Kontext betrieblicher Personalpolitik. Soz. Welt 61(1), 2750 (2010)

Hohendanner, C., Janik, F.: Praktika und betriebliche Personalpolitik. Verbreitung und Nutzungsintensität von Praktika in deutschen Betrieben. Z. Arb.markt Forsch. 41(4), 471-487 (2008)

Horbach, J.: Beschäftigungserwartungen und Innovationen im Umweltbereich. Eine empirische Analyse auf der Basis des IABBetriebspanels. Mitt. Arb.markt- Berufsforsch. 36(3), 291-299 (2003)

Horbach, J.: The impact of innovation activities an employment in the environmental sector. Empirical results for Germany at the firm level. Jahrb. Natl.ökon. Stat. 230(4), 403-419 (2010)

Hübler, O.: Sind betriebliche Bündnisse für Arbeit erfolgreich? Jahrb. Natl.ökon. Stat. 235(6), 630-652 (2005)

International Labour Office: Technical Meeting on Enterprise Restructuring and Labour Markets, Konferenzbeiträge, Turin, 31. Mai bis 02. Juni (1995)

Kohaut, S., Schnabel, C.: Zur Erosion des Flächentarifvertrags. Ausmaß, Einflussfaktoren und Gegenmaßnahmen. Ind. Bez. 10(2), 193-219 (2003)

Kohlmann, A.: The research data centre of the federal employment service in the institute for employment research. Schmollers Jahrb. Wirtsch.- Soz.wiss. 125(3), 437-447 (2005)

Kölling, A.: The IAB-establishment panel. Schmollers Jahrbuch Z. Wirtsch.- Sozialwiss. 120(2), 291-300 (2000)
Kölling, A., Möller, I.: Betriebliche Determinanten innovativer ,work practices" und ihre Auswirkungen auf die Beschäftigtenstruktur. In: Bellmann, L., Kölling, A. (Hrsg.) Betrieblicher Wandel und Fachkräftebedarf. Beiträge zur Arbeitsmarkt- und Berufsforschung, Bd. 257, S. 173-198 (2002). Nürnberg

Kölling, A., Schank, T.: Skill-biased technological change, international trade and the wage structure. New evidence on the determinants of the employment structure from linked employeremployee panel data for Germany. In: Tenth Conference on Panel Data, Aarhus, 6. und 7. Juli 2002 (2002)

Kropp, P., Schwengler, B.: Abgrenzung von Arbeitsmarktregionen ein Methodenvorschlag. Raumforsch. Raumordn. 69(1), 45-62 (2011)

Krugman, P.: Past and prospective causes of unemployment: economic review. Federal Reserve Bank of Kansas City, QIV, S. 23-43 (1994)

Krugman, P.: Free to loose. New York Times 12.11.2009 (2009)

MEADOWS-Projekt: The Meadows Guidelines. (2010). Brüssel

Moritz, M., Hecht, V.: Warum deutsche Unternehmen in Tschechien investieren. IAB-Forum 1/2013, 73-81 (2013)

Möller, I.: Produktivitätswirkung von Mitarbeiterbeteiligungen. Mitt. Arb.markt- Berufsforsch. 33(4), 565-582 (2000)

Möller, J.: The German labor market response in the world recessionde-mystifying a miracle. J. Labour Mark. Res. 42, 325-336 (2010)

Münich, D., Srholec, M., Moritz, M., Schäffler, J.: Mothers and daughters: heterogeneity of German direct investments in the Czech Republic. Evidence from the IAB-ReLOC survey. IAB discussion paper 17/2012. Institut für Arbeitsmarkt und Berufsforschung, Nürnberg (2012)

Niederalt, M.: Zur ökonomischen Analyse betrieblicher Lehrstellenangebote in der Bundesrepublik Deutschland. Europäische Hochschulschriften. Reihe 5, Volks- und Betriebswirtschaft, Bd. 3082 (2004). Frankfurt am Main

OECD: Labour Market Flexibility. Trends in Enterprises. (1989). Paris

OECD: The OECD Jobs Study. Evidence and Explanations. Part I. Labour Market Trends and Underlying Forces of Change. Part II. The Adjustment Potential of the Labour Market (1994). Paris

OECD: Oslo Manual: Guidelines for Collceting and Interpreting Innovation Data (2005). Paris

OECD: What makes labour markets resilient during recessions? In: OECD Employment Outlook 2012, S. 53-107. OECD, Paris (2012). Chap. 2

Pflüger, M., Blien, U., Möller, J., Moritz, M.: Labor market effects of trade and FDI: recent advances and research gaps. Jahrb. Natl.ökon. Stat. 233(1), 86-116 (2013)

Promberger, M.: Leiharbeit - Flexibilität und Prekarität in der betrieblichen Praxis. WSI-Mitt. 56(5), 263-269 (2006)

Rabe-Hesketh, S., Skrondal, A.: Multilevel and Longitudinale Modeling Using Stata, Vols. I + II. Stata Press, Texas (2012)

Roubini, N.: Crisis Economics. A Crash Course in the Future of Finance. (2010). London

Schank, T.: The Impact of Working Time on Employment, Wages and Productivity. Evidence from IAB Establishment Panel Data. Beiträge zur Arbeitsmarkt- und Berufsforschung, Bd. 269 (2003). Nürnberg

Schank, T., Schnabel, C., Gerner, H.-D.: Stimulating part-time work by legal entitlements? Evidence from a German policy experiment. Appl. Econ. Lett. 16(4), 391-394 (2009)

Stiglitz, J.: The global crisis, social protection and jobs. Int. Labour Rev. 148, 1-14 (2009)

Wolf, E., Zwick, T.: Produktivitätswirkung von Mitarbeiterbeteiligung. Der Einfluss von unbeobachteter Heterogenität. Mitt. Arb.marktBerufsforsch. 35, 123-132 (2002)

Wolf, E., Zwick, T.: Reassessing the productivity impact of employee involvement and financial incentives. Schmalenbach Bus. Rev. 60(2), 160-181 (2008) 
Womack, J.P., Jones, D.T., Roos, D.: Die zweite Revolution in der Automobilindustrie (1991). Frankfurt a.M./New York

Prof. Dr. Lutz Bellmann leitet sowohl das IAB-BetriebspanelProjekt als auch den Forschungsbericht „Betriebe und Beschäftigung“. Seit 2009 hat er auch einen Lehrstuhl für Volkswirtschaftslehre insbesondere Arbeitsökonomie an der Friedrich-Alexander-Universität Erlangen-Nürnberg inne. Er erwarb sein Diplom 1980 in Wirtschaftswissenschaften an der Universität Hannover. Dort promovierte er 1985,
2004 erlangte er die Habilitation. Prof. Bellmann hat in internationalen Journals wie International Journal of Manpower, Industrial Relations, Journal of Applied Social Science Studies und dem British Journal of Industrial Relations publiziert. Arbeits- und Bildungsökonomische Studien zu Fragen der industriellen Beziehungen, den Determinanten der Beschäftigung, betriebliche Aus- und Weiterbildung sowie atypische Beschäftigung bilden den Schwerpunkt seiner Forschungstätigkeit. e-mail: Lutz.Bellmann@iab.de 